\title{
Characterization of human persistent atrial fibrillation electrograms using recurrence quantification analysis
}

Tiago P Almeida, $\mathrm{PhD}^{1,2}$; Fernando S Schlindwein, $\mathrm{PhD}$, DSc ${ }^{3,4}$; João Salinet, $\mathrm{PhD}^{2}$; Xin $\mathrm{Li} \mathrm{PhD}^{5}$; Gavin

S Chu, MB BChir, MA(Cantab), MRCP(UK) ${ }^{5,6}$; Jiun H Tuan, MB BS, MD, MRCP ${ }^{6}$; Peter J Stafford, MB BS, MD, FRCP ${ }^{6}$; G André Ng, MBChB, PhD, FRCP(Glasg), FRCP, FESC ${ }^{4,5,6}$; Diogo C. Soriano, PhD ${ }^{2}$

${ }^{1}$ Aeronautics Institute of Technology, ITA, Brazil; ${ }^{2}$ Engineering, Modelling and Applied Social Sciences Centre, Federal ABC University, Brazil; ${ }^{3}$ Department of Engineering, University of Leicester, UK; ${ }^{4}$ National Institute for Health Research Leicester Cardiovascular Biomedical Research Centre, Glenfield Hospital, UK; ${ }^{5}$ Department of Cardiovascular Sciences, University of Leicester, UK; ${ }^{6}$ University Hospitals of Leicester NHS Trust, UK.

\section{Address for correspondence:}

Dr. Diogo C. Soriano, Federal ABC University, Brazil

Alameda da Universidade

São Bernardo do Campo, SP, CEP 09606-045, Brazil

Email: diogo.soriano@ufabc.edu.br

Tel: $+55(11) 23206342$

Total number of words of the manuscript: 5161 (excluding abstract, title, author list, references, and acknowledgments)

Number of words of the abstract: 246

Number of figures: 7

Number of tables: 3 


\begin{abstract}
Atrial fibrillation (AF) is regarded as a complex arrhythmia, with one or more co-existing mechanisms, resulting in an intricate structure of atrial activations. Fractionated atrial electrograms (AEGs) were thought to represent arrhythmogenic tissue, and hence have been suggested as targets for radiofrequency ablation. However, current methods for ablation target identification have resulted in suboptimal outcomes for persistent AF (persAF) treatment, possibly due to the complex spatiotemporal dynamics of these mechanisms. In the present work, we sought to characterize the dynamics of atrial tissue activations from AEGs collected during persAF using recurrence plots (RPs) and recurrence quantification analysis (RQA). 797 bipolar AEGs were collected from 18 persAF patients undergoing pulmonary vein isolation (PVI). Automated AEG classification (normal vs. fractionated) was performed using the CARTO criteria (Biosense Webster). For each AEG, RPs were evaluated in a phase space estimated following the Takens' theorem. Seven RQA variables were obtained from the RPs: recurrence rate; determinism; average diagonal line length; Shannon entropy of diagonal length distribution; laminarity; trapping time; and Shannon entropy of vertical length distribution. The results show that the RQA variables were significantly affected by PVI, and that the variables were effective in discriminating normal vs. fractionated AEGs. Additionally, diagonal structures associated to deterministic behavior were still present in the RPs from fractionated AEGs, leading to a high residual determinism, which could be related to unstable periodic orbits and suggesting a possible chaotic behavior. Therefore, these results contribute for a nonlinear perspective of the spatiotemporal dynamics of persAF.
\end{abstract}

Keywords: Persistent atrial fibrillation; fractionated electrograms; catheter ablation; electrophysiology mapping; recurrence plots, recurrence quantification analysis 
Lead paragraph

Biological markers that better explain atrial fibrillation (AF) behavior and provide a definitive answer for persistent atrial fibrillation (persAF) therapy are still in debate due to its complex underlying pathophysiology and spatiotemporal behavior. As such, the role of low dimensional structures for explaining AF has been the subject of many investigations, showing that recurrence quantification analysis (RQA) might be useful to explore the underlying AF dynamics. However, a consistent set of RQA variables taking into account the specificities of the signals and of the theoretical methodology are still needed. In the present work, we propose rigorous steps for a proper reconstruction of the recurrence plots (RPs) and for the estimation of RQA-based variables extracted from atrial electrograms (AEGs) collected from persAF patients undergoing a clinical procedure for AF therapy. We demonstrate that these RQA-based variables are sensitive to important electrophysiologic characteristics of the atrial tissue, and could be potentially used as biological markers to guide the clinical procedure. Additionally, a high residual determinism was found in the RPs from AEGs with seemingly turbulent characteristics, which implies that the spatiotemporal dynamics of persAF mechanisms is not necessarily associated to a random structure. 


\section{Introduction}

The dynamics of cardiac signals in the presence of arrhythmias have been extensively investigated in the context of recurrence analysis, especially when considering the main advantages of recurrence plots (RPs) and recurrence quantification analysis (RQA) for characterizing short time series, phase transitions, nonstationarity and unveiling nonlinear underlying phenomena in general [1-7]. The possibility of quantifying i) signal regularity, laminarity and determinism; ii) nonlinear topological invariants - e.g., correlation dimension and Kolmogorov-Sinai entropy $[8,9]-$ iii) information-theoretic measures - e.g., generalized entropies [8] - and; iv) mutual information [3,10,11], outlines the RQA properties as a singular framework for cardiac analysis in wide sense over more traditional methods $[2,4,7,12,13]$.

Investigations regarding the dynamical behavior of cardiac fibrillation have resulted in tantalizing discussions on whether sustained arrhythmias could be better explained by a deterministic model (i.e., free of probability density functions) or by a stochastic approach. While evidences suggesting possible chaotic dynamics - i.e., described by a set of nonlinear differential equations providing an aperiodic and phase space compact solution trajectory sensible to initial conditions - have established an important theoretical framework [14-16], studies concerning power spectrum [17] and correlation dimension estimation from fibrillation in dogs [18] have failed to detect such chaotic behavior and a low dimensional phase space explanation for the observations.

It is important to emphasize, however, that the experimental detection of chaotic phenomena usually exhibits drawbacks as a consequence of additive (measurement) noise, unstable experimental conditions and the requirements for a large amount of data for suitable statistical characterization as occurs, for instance, in the classical Grassberger \& Proccacia (GP) algorithm for correlation dimension estimation [19]. These drawbacks motivate the employment of different strategies for characterizing the fibrillation behavior and its possible nonlinear nature and justify the use of RQA due to its intrinsic features previously mentioned. 
Studies on atrial fibrillation (AF) are of particular interest. AF is the most common sustained arrhythmia in clinical practice, and is a leading cause of hospitalization and cardiovascular complications, particularly stroke [20]. It is defined as a supraventricular tachyarrhythmia characterized by uncoordinated atrial activation with consequent deterioration of atrial mechanical function [20]. Radiofrequency catheter ablation is considered the cornerstone for AF percutaneous therapy. Success rates as high as $90 \%$ have been reported after pulmonary vein (PV) isolation (PVI) through ablation in patients with paroxysmal AF [20, 21]. For the treatment of persistent AF (persAF), however, PVI has been shown insufficient due to, among others, extensive atrial remodeling induced by sustained AF that, in turn, also participate directly in the perpetuation of the arrhythmia [22].

Different mechanisms are likely to participate in persAF perpetuation linked to remodeled substrate, such as the rapidly discharging automatic foci [21]; the multiple wavelets hypothesis [23]; the single reentrant circuit with fibrillatory conduction [24]; the conduction dissociation between epicardial and endocardial layers [25]; and functional reentry resulting from rotors [26]. Biological markers have been introduced in attempts to characterize the atrial substrate and guide persAF ablation, with promising initial results [2628]. Subsequent investigations, however, could not reproduce such outcomes, and the search for markers that better explain AF behavior to guide persAF ablation are still one of the most debated topics in AF studies [29-36]. Fractionation in AEGs collected during persAF are of particular interest [27]. During persAF, fractionated activity has been linked to: i) random activations from meandering wavelets that propagate through the atria; ii) underlying anisotropic conduction in the atrial remodeled tissue and iii) the occurrence of wave breaks or wave collisions in the atrial tissue [37]. Commercial systems are still broadly used for automated AEG classification - normal vs. fractionated - for target identification during persAF ablation (Figure 1), also with inconsistent outcomes [37].

Current markers for ablation might have failed to provide a definitive solution for persAF therapy possibly because they have been insufficient to fully describe the complex AF dynamics. Recent works have highlighted the unstable spatiotemporal behavior of underlying atrial activations during persAF, 
with AF drivers that drift over the left atrium (LA) [38-41]. The same works, however, have shown that these drivers have preferential sites for recurrence. More recently, the nature of AF has been described as a spatiotemporally chaotic state linked to the alternans instability that leads to conduction block and wave breakup, which supports recent findings regarding drivers recurrence in preferential atrial regions [42].

The role of low dimensional structures for explaining AF has also been the subject of many investigations. In a seminal work, Hoekstra and colleagues have detected convergent low dimension trajectories for AF type I - as defined by Wells et al. [43] - using correlation dimension estimation by means of GP algorithm, while failing to find such finite scaling structure for types II and III, which may also be associated to the GP algorithm limitations [19]. In the context of RP and RQA, Censi and colleagues have analyzed recurrent patterns on atrial activations in different atrial regions from paired sets of AEGs collected simultaneously, suggesting a theoretical paradigm for atrial functional coupling [44]. More recently, Navoret and colleagues have used RQA-based variables in the classification of AEGs and investigated the temporal dynamics of AEGs during AF, without, however, describing deterministic structures from the AEGs [45]. Ng et al. have used a modified concept of RPs to investigate the regularity of segmented activations in the AEGs, which stablished an interesting recurrence-based activation map associated to the study of specific waveforms propagation $[46,47]$. Zeemering and colleagues have used RQA to investigate the spatiotemporal pattern of AEGs using high-density mapping [48]. In the latter, RQA-based variables and principal component analysis were used, among others, to correlate RQA's determinism and the number of waves per AF cycle, providing an alternative measure associated to the complexity of the arrhythmia. Finally, Hummel et al. have introduced a method for quantifying recurrent patterns of local wavefront direction using RQA and the conformation of AEGs during AF [49]. The authors have shown that the RPs from areas of stable spiral wave reentry exhibited stable, periodic dynamics, while regions of wavelet breakup have shown a chaotic behavior largely devoid of repetitive activation patterns. 
The nature of this problem, therefore, suggests that recurrence analysis might be useful to explore the underlying AF dynamics, and may provide additional information about the underlying atrial substrate, potentially helping in the identification of targets for persAF ablation. These require the definition of a consistent set of RQA variables taking into account the specificities of the signals and of the theoretical methodology. In the present work, we sought to quantify the changes in AF dynamics induced by PVI as measured by RQA variables in patients with persAF. A comparison between normal and fractioned AEGs was then performed in the RQA-variables domain considering the clinical condition (label) defined by the CARTO criteria (Biosense Webster, Diamond Bar, California) - a commercial system broadly used for automated AEG classification and ablation target identification. To accomplish such RQA characterization of $\mathrm{AF}$, we proposed an adaptive threshold distance $\varepsilon$ for recurrence definition considering different criteria in the literature, as also the typical high laminarity observed in normal AEGs, and the AEG discrimination based on CARTO criteria.

\section{Methods}

\section{a. Study population and electrophysiological Study}

The population consisted of 18 patients (16 male; mean age $56.1 \pm 9.3$ years; history of AF $67.2 \pm 45.6$ months) referred to our institution for first time catheter ablation of persAF [50]. Details of the clinical characteristics of the study subjects are provided in the Supplementary Material. All patients were in AF at the start of the procedure. Study approval was obtained from the local ethics committee and all procedures were performed with full informed consent.

All antiarrhythmic drugs, except amiodarone, were discontinued for at least 5 half-lives before the start of the procedure. Details of the mapping procedure have been described previously [50]. Briefly, 3D LA geometry was created within NavX ${ }^{\mathrm{TM}}$ (St. Jude Medical, St. Paul, Minnesota) using a deflectable, variable loop circular PV mapping catheter (Inquiry Optima, St. Jude Medical) (Figure 2A). PVI was performed with a point-by-point wide area circumferential ablation approach (Cool Path Duo irrigated RF catheter, 
St. Jude Medical), followed by the creation of a single roof line (RL). PVI was defined as the abolition of electrical signals on the circular mapping catheter when positioned within each PV.

No additional ablation targeting fractionated AEGs was performed in this study. AEG collection was performed for further offline analyses. Sequential point-by-point bipolar AEGs were collected also using the Inquiry Optima from 15 pre-determined atrial regions before and after PVI and RL creation (PVI+RL) [50]. All patients were in AF before and after PVI+RL during signal collection.

\section{b. Signal analysis}

A total of 797 AEGs were recorded from the LA, 455 before and 342 after PV+RL (1200 Hz sampling frequency; 30-300 Hz band-pass filter built in the NavX system [37]; $50 \mathrm{~Hz}$ Notch filter) (Figure 2B). A stationary wavelet transform (SWT) filter was implemented based on a previously described method to further reduce both baseline oscillations and high frequency noise [51]. For baseline oscillations, the AEGs were decomposed with Daubechies D11 wavelet into details 8, corresponding to the frequency band between $0-2.34 \mathrm{~Hz}$, which was set to zero. For the high frequency noise, the AEGs were decomposed with Haar wavelet into details 7. Level 1 corresponds to frequency band between $300-600$ $\mathrm{Hz}$, which has no electrophysiologic relevance. Hence, it was assumed that the presence of a white noise - that affects the frequency spectrum homogeneously - would be more evident in this frequency band with variance $\sigma_{w}^{2}$. An adaptive threshold was calculated for each AEG, accordingly:

$$
T_{r}=v_{W}^{2} \sqrt{2 \cdot \ln N}
$$

where $N$ is the length of the AEG. The threshold $T_{r}$ represents the amplitude level of the white noise distributed in the AEG. This threshold was then applied in all the levels of the filter bank. At each level, amplitudes higher than the threshold were conserved, while amplitudes below the threshold were suppressed. The resulting filtered AEGs were computed with the levels after thresholding with the inverse wavelet transform. 


\section{c. The CARTO algorithm (CARTO 3 System, 2008-2014, Version 4.3)}

AEG classification (normal vs. fractionated) was performed following the CARTO criteria. A detailed description of the CARTO system is provided elsewhere [37]. Briefly, CARTO provides a 3D representation of the LA and online automated AEG classification based on complex intervals between successive peaks and troughs occurring inside a 2.5 seconds window of sequentially recorded bipolar AEGs (Figure 1). The number of identified complex intervals is referred to as the interval confidence level (ICL), and characterizes the repetitiveness of the fractionated complexes, which is then converted to a color-coded map that guides ablation. CARTO software also finds, as complementary attributes, the average of the identified interval, referred to as the average complex interval (ACI), and the shortest identified interval, referred to as the shortest complex interval (SCI). AEGs were considered fractionated for $\mathrm{ICL} \geq 4, \mathrm{ACI} \leq 82 \mathrm{~ms}$ and $\mathrm{SCI} \leq 58 \mathrm{~ms}[37]$.

Therefore, AEGs with high amplitude, discrete activations distanced by electrical silence (such as AEG 1 in Figure 1) are considered normal AEGs, and are believed to represent healthy atrial tissue. AEGs with low amplitude, multiple deflections activations (such as AEG 2 in Figure 1) are considered fractionated AEGs, and are believed to represent atrial substrate.

\section{d. Recurrence quantification analysis}

A RP consists of a two-dimensional graphical technique used for the study of the topological structure of multidimensional dynamic systems [3]. It aims to evaluate the reappearance of states in a multidimensional vector based on the construction of a binary distance matrix, associating the value " 1 " whenever one point in the multidimensional vector is close to another sample by a distance less than $\varepsilon$, and "0", otherwise. This recurrence matrix can be represented graphically by associating a black pixel to the value "1". This binary signal "fingerprint" exhibited in the RP is related to its dynamics allowing access to stationarity, regularity and predictability, being useful to characterize phase transitions and the temporal evolution in general, even considering a small amount of data samples, since information 
concerning the distance organization between all pair of points is taken into account in time and phase space.

Complex patterns in RPs can be reduced to primary diagonal, vertical and horizontal dot patterns aligned on a grid [52]. It is the mixing and matching of these primary structures that gives rise to all derivative graphical complexities and allows for the quantification of the recurrence organization. Therefore, RQA was established by introducing statistical variables considering the distribution of diagonal and vertical lines of different sizes in the RP [53]. Since usually just a single observation (the AEG) is available for a time window, the phase space can be reconstructed using the Takens' theorem [3], aiming to reveal a possibly hidden low dimensional geometric trajectory underlying the observation, as shown in Figure 2C. In this case, the auxiliary axes are estimated considering delayed samples of the unidimensional observation $\mathbf{x}(k)$, such as:

$$
\mathbf{x}(k)=\left[x(k)_{r} x(k-\tau)_{r} \boldsymbol{n}_{t} x\left(k-\left(d_{\varepsilon}-1\right) \tau\right)\right]
$$

in which $d_{e}$ is the embedding dimension - number of coordinates used for the $\mathbf{x}(k)$ representation - and $\tau$ represents the time-delay embedding among samples. These parameters were estimated through the false nearest neighbor test and the first local minimum of the self-mutual information, respectively, as classically performed in nonlinear analysis devoted to RP and RQA [3, 54].

The recurrence matrix $\left(\mathrm{R}_{i, j}\right)$ was defined based on the distance between the respective reconstructed states $\mathbf{x}_{i}$ and $\mathbf{x}_{j}$ and an arbitrary threshold $\varepsilon$ for defining a binary proximity relation, which can be expressed as:

$$
\mathrm{K}_{i, j}(s)=\Theta\left\{s-\left\|\mathrm{x}_{i}-\mathrm{x}_{i}\right\|\right\}
$$

being $\theta\{\cdot\}$ the Heaviside function (Figure 2D).

The binary pattern observed in a RP is intrinsically related to the generative dynamics of the observation: the horizontal structures are associated with the laminarity (or turbulence) of the signal; the diagonal 
structures reflect the co-evolution of states, and indicate possible deterministic dynamics. For a RP obtained from a time series of $N$ samples, with frequency distribution $\mathrm{P}(l)$ of diagonals line length, frequency distribution $\mathrm{P}(v)$ of vertical line length, probability $\mathrm{p}(l)$ of finding a diagonal of length $l$, probability $\mathrm{p}(v)$ of finding a vertical line of length $v$, the RQA is associated with representative statistical measures of the matrix $\mathrm{R}_{i, j}[3]$, being used here:

- The recurrence rate (RR), which represents the density of points in a RP:

$$
R R=\frac{1}{N^{2}} \sum_{t h=1}^{N} R_{t h}
$$

- The ratio between recurrence points that form diagonal structures (with length $l \geq l_{\min }$ ), referred to as determinism (DET):

$$
D E Y=\frac{\sum_{i=i_{\min }}^{W Q} l \mathrm{P}(i)}{\sum_{i=1}^{W} l \mathrm{P}(l)}
$$

- $\quad$ The average length of the diagonal lines (L):

$$
L=\frac{\sum_{i=i_{\min }}^{W} l \mathrm{P}(l)}{\sum_{i=i_{\min }}^{W} \mathrm{P}(l)}
$$

- The Shannon entropy of diagonal lines in a RP (ENTR), which reflects the RP's complexity regarding the diagonal lines:

$$
E N T R=-\sum_{i=i_{m i n}}^{N} p(l) \ln p(l)
$$

- The ratio between recurrence points that form vertical structures (with length $v \geq v_{\text {min }}$ ), referred to as laminarity (LAM): 


$$
\operatorname{LAM}=\frac{\sum_{v=v_{\min }} v \mathrm{P}(v)}{\sum_{v-1}^{N} \tau \mathrm{P}(v)}
$$

- The trapping time (TT), which represents the average length of the vertical lines:

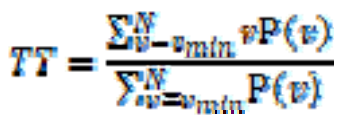

- The Shannon entropy of vertical lines in a RP (ENTR_vert), which reflects the RP's complexity regarding the vertical lines:

$$
\text { ENTE_vert - - } \sum_{v=w_{\min }}^{N} p(v) \ln p(v)
$$

These RQA-based variables were investigated for AEGs collected before and after PVI+RL. Comparisons were also conducted on these RQA-based values calculated from normal vs. fractionated AEGs.

\section{e. Defining \&-parameters for $R Q A$}

After embedding dimension definition by nearest neighbors' algorithm and setting $\tau$ based on minimal mutual information, $\varepsilon$ was determined. Previous work has suggested that $\varepsilon$ should be chosen such that the resulting RR is approximately $1 \%$, or $\varepsilon$ should not exceed $10 \%$ of the maximum phase space diameter [3]. Therefore, eleven different values for $\varepsilon$ were tested, varying from $0.5 \%$ to $10 \%$ of the maximum phase space diameter. For each $\varepsilon$, the RR was calculated, and the discrimination between normal $v s$. fractionated AEGs was tested using the MATLAB ${ }^{\circledR}$ embedded function rankfeatures ('CriterionValue' set at 'roc'). Similarly, the minimum line lengths for the calculation of DET and LAM were defined considering the discrimination between normal vs. fractionated AEGs using the MATLAB ${ }^{\circledR}$ embedded function rankfeatures ('CriterionValue' set at 'roc'). Using the $\varepsilon$ defined above, both DET and LAM were calculated for nineteen values of minimum line length, varying from 2 to 20 . The minimum line lengths 
were also defined as a compromise between the resulting values for DET and LAM, and AEG discrimination. The determination of $\varepsilon$ has also taken into account the fact that normal AEGs are usually quite regular (as shown in Figure 1) and exhibit high laminarity as an intrinsic behavior, which would to contribute for a high RR and far from the suggested $1 \%$ rule. The proposed approach aimed to choose an adaptive $\varepsilon$ as a percentage of maximal phase space difference, leading to a relatively low RR and distinguishable between normal and fractionated AEGs pointed by CARTO criteria in the DET and LAM variables.

\section{f. Statistical Analysis}

All continuous non-normally distributed variables are expressed as median \pm interquartile interval. Nonparametric unpaired data were analyzed using the Mann-Whitney test. The comparison between two probability distributions was carried with the Kullback-Leibler (KL) divergence. Receiver operating characteristic (ROC) curves were created using the CARTO criteria as the reference for AEG classification and the RQA variables as discriminators. The optimum sensitivity and specificity on the ROC curve was defined as the point on the curve with the shortest distance to the top left corner of the graph. Validation of the optimum point of operation for AEG classification was performed with leaveone-out cross-validation (LOOCV). P-values of less than 0.05 were considered statistically significant.

\section{Results}

\section{a. Variables for $R Q A$}

For the calculation of $\varepsilon$, the area under the ROC (AUROC) curves - shown in Table 1 - suggest that 2\% of the maximum phase space diameter represents a good compromise among the resulting RR, the discrimination between normal and fractionated AEGs (Figure 3A), and the portion of the maximum phase space diameter. Similarly, the AUROC curves shown in Table 2 suggest that a minimal line length of 6 should be considered for the calculation of DET (Figure 3B), while a minimal line length of 7 should be considered for the calculation of LAM (Figure 3C) for a high class discrimination and also minimal 
and maximal bounds to the attributed estimates. Note that minimal vertical or diagonal lengths of 2 or 3 can lead to maximal DET or LAM, which may be associated to an undesirable saturation behavior. Additionally, Figures 3B and 3C also show that the percentage of vertical and diagonal lines in longer lengths are non-negligible even for fractionated AEGs, illustrating the changes in both DET and LAM induced by the minimal line length parameter expressed in Eqs. (5) and (8).

These values were used for the construction of the RPs, and the calculation of DET and LAM for each AEG in the subsequent parts of this work.

\section{b. Filtering effect on RQA}

The effect of the SWT filter has been investigated (Figure 4). The SWT filter helped to remove unwanted low amplitude, high frequency components. Consequently, the RP created from filtered AEG was represented by blocks of recurrence due to the laminar nature of the signal, while the unfiltered AEGs represented a blurred version of such RP (Figure 4A). This was supported by the evident effect on the histogram of the diagonal line lengths illustrated in Figure 4B, showing that lines of length 1 (related to noise) were drastically reduced, while the lines with longer lengths remained mostly unaffected. Despite this impact, the KL divergence suggested a small difference between the two distributions (unfiltered $v s$. filtered AEGs).

\section{c. Atrial substrate characterization using RQA-based variables}

Two typical AEGs - one normal and one fractionated - found in the present cohort and their respective RPs are illustrated in Figure 5. While normal AEGs were characterized by evident blocks of recurrence and longer diagonal lines, reflecting the laminar and deterministic behavior of the system, fractionated AEGs have presented low RR, but with residual diagonal lines.

The comparison of RQA-based variables between normal and fractionated AEGs is shown in Figure 6. All variables were significantly affected by the presence of fractionated activity $(\mathrm{P}<0.0001$ for all cases $)$. 
As expected, RR was lower for fractionated AEGs (Figure 6A). However, the variables related to the diagonal lines in the RPs (DET, L and ENTR) suggest the presence of structures that are associated to deterministic behavior in fractionated AEGs, leading to a high residual determinism (Figure 6B). Similarly, the variables related to the vertical lines (LAM, TT and ENTR_vert) also suggest the presence of vertical structures associated to laminar behavior in the fractionated AEGs, leading to a residual laminarity (Figure 6C). Additionally, the variables were able to discriminate normal AEGs from fractionated ones, as illustrated by the ROC curves (supplementary materials), with a hit rate from the LOOCV as high as 69\% (Table 3).

\section{d. Effect of PVI+RL on the RQA-based variables}

The effect of PVI+RL on the RQA-based variables is shown in Figure 7. All features were significantly affected by the procedure $(\mathrm{P}<0.0001$ for all cases), suggesting that they are sensitive to important electrophysiologic characteristics of the atrial tissue. PVI+RL increased the RR, suggesting that the ablation evidenced periodic orbits in the LA (Figure 7A).

The variables related to the diagonal lines indicate that PVI+RL increased the overall diagonal line lengths - related to deterministic structures (Figure 7B). Additionally, higher ENTR reflects the increase of the dispersion in the probability distribution of the diagonal line lengths induced by PVI+RL. Altogether, these results suggest that the RPs from the AEGs collected at baseline presented a high incidence of diagonal lines with short lengths, while the ablation increased both the lengths and the variance of the probability distribution of these diagonal lines.

Similarly, the variables related to the vertical lines indicate that PVI+RL increased the overall vertical line lengths - related to the system's laminarity (Figure 7C). Additionally, higher ENTR_vert reflects the increase of the dispersion in the probability distribution of the vertical line lengths induced by PVI+RL. These results also suggest that the RPs from the AEGs collected at baseline presented a high incidence of 
vertical lines with short lengths, while the ablation increased both the length and the variance of the probability distribution of these vertical lines.

Hence, PVI+RL had a profound effect in the dynamics of the underlying atrial tissue, and the AEGs collected after the ablation were more organized - and therefore less fractionated - than those collected at baseline.

\section{Discussion}

In this work, we further investigated the dynamic structure of AEGs collected during persAF using RQA following the automated AEG classification performed by CARTO. The results show that residual diagonal structures associated to deterministic behavior were still present in the RPs from fractionated AEGs, leading to a high residual determinism, which could be related to unstable periodic orbits in a chaotic motion. This implies that the spatiotemporal dynamics of persAF mechanisms are not necessarily associated to a random structure $[42,55]$. On the contrary, even the seemingly turbulent activations in fractionated AEGs are related to deterministic mechanisms of activation that could be a result of different AF drivers in the LA responsible for triggering and maintaining AF $[39,41,56]$. Therefore, RQA could be potentially used to better characterize the atrial substrate during persAF and identify targets for ablation.

\section{a. RQA-based variables from pers $A F$ AEGs}

In the present study, we propose rigorous steps for a proper reconstruction of the RPs and for the estimation of RQA-based variables extracted from persAF AEGs. First, the AEGs have been filtered using SWT filter banks. This represents an important step in the experimental characterization and identification of chaotic behavior. Proper AF characterization requires the removal of additive noise, and the SWT denoising has been shown effective to achieve that [57]. The additive components removed from AEGs were, in general, ten times smaller than the remaining signal, in amplitude. This resulted in the removal of mostly single isolated points in the RP - as shown in Figure 4 - and did not introduce 
distortions on longer diagonals, as would be associated to an undesirable increase of determinism. Interestingly, classical low pass filtering has been shown to distort the deterministic dynamics [58], which seems to be avoided in the present work using SWT denoising strategy.

Following the SWT filtering, the Takens' theorem was applied for the estimation of the attractors for all AEGs, in which the false nearest neighbor test and the self-mutual information were used for the estimation of the embedding dimension and the time-delay embedding, respectively $[3,54]$.

A thorough investigation was then conducted to better define $\varepsilon$ for each AEG. Previous work has shown the importance of finding $\varepsilon$ as such the resulting RR should converge to $1 \%$ [3], but the high laminar nature of the AEGs would limit this application. Therefore, an adaptive value for $\varepsilon$ has been estimated in order to achieve a resulting $\mathrm{RR}$ as close to $1 \%$ as possible, but also with a good compromise between the AEG discrimination and the portion of the maximum phase space diameter.

The minimum line length for the calculation of both DET and LAM has been also assessed. The results suggest that DET and LAM are preserved even for different minimum line lengths. In this work, DET and LAM were calculated considering minimum line lengths of 6 and 7, respectively, to avoid undesirable saturation behavior and for improved AEG discrimination.

Finally, the AEGs have been filtered using an adaptive SWT filter in an attempt to minimize additive (measurement) noise. The filter succeeded in removing a significant portion of diagonal lines with length one, i.e., recurrent components very likely associated with stochastic phenomena.

\section{b. Pers AF AEG characterization using RQA}

Previous works have considered RPs and RQA for the investigation of AF dynamics [44-49]. Our work represents a step further in the attempt to i) assess changes in RQA-based variables induced by PVI; ii) evaluate AEG classification using RQA-based variables considering a clinical label as reference and; iii) 
unveil possible deterministic structures within the temporal series from single AEGs using the Takens' theorem to estimate the underlying attractor.

Although Zeemering and colleagues have considered electrophysiologic criteria instead of the Takens' theorem for the estimation of the attractors [48], the authors have provided great insight on the correlation between RQA-based variables and the AF electrophysiology - there represented by the existing number of wavelets during one activation episode. In the present work, the AF electrophysiology was assessed by RQA-based variables considering the PVI - the most accepted and broadly performed percutaneous therapy for AF treatment [21]. Statistically significant shifts in the RQA attributes were discovered after PVI (Figure 7), which indicates these attributes are related to important electrophysiological changes in the arrhythmia. While this does not provide any predictions as to where to ablate, we believe it is imperative to understand the changes (if any) in these variables after PVI prior to any further analysis considering that: i) this is as an exploratory step for persAF characterization using RQA-based variables and; ii) it is known that PVI induces changes in the atrial substrate, even in regions far from ablation lesions [29].

Furthermore, statistically significant differences were discovered in the RQA variables when comparing normal AEGs vs. fractionated AEGs (Figure 6) - in line with previously published data [48]. The high residual determinism from these seemingly turbulent (or fractionated) activations could be related to unstable periodic orbits in a chaotic motion [42]. Therefore, the spatiotemporal dynamics of persAF mechanisms were not associated to a random structure. Accordingly, recent works have highlighted the unstable - yet recurrent - behavior of underlying atrial activations during persAF [38-41]. Rotors have been shown to be spatiotemporally unstable, appearing and disappearing in different atrial regions [39, 40]. The same works, however, have shown that, although reentry circuits meander in the atria, they have preferential sites for recurrence. Additionally, a recent published work has shown a high correlation between the recurrence of reentry circuits and atrial regions with high fibrosis density [59]. Frontera and colleagues have recently reported fractionated activity from the pivot points of reentry circuits [60]. 
Finally, Hummel et al. have shown that the RPs from areas of stable spiral wave reentry exhibited stable, periodic dynamics, while regions of wavelet breakup have shown a chaotic behavior largely devoid of repetitive activation patterns [49]. These results suggest that fractionated activity could be related to areas of high density of fibrosis that would induce the formation of reentry circuits, from which multiple wavelets might emanate and meander in the atrial tissue, with direct participation in the perpetuation of the arrhythmia.

Although fractionation-guided ablation is one of the most controversial topics in patient-specific AF therapy [37], these findings motivate further investigation as to use RQA variables as biological markers that can potentially guide persAF ablation in the future. Hence, our results support that the RQA-based variables not only provide further insight on the underlying dynamics of the arrhythmia [49], but they might also be used as complementary biological markers in the characterization of atrial substrate to guide persAF ablation.

\section{c. Limitations}

The current study was limited to retrospective data. Further understanding of the underlying cardioelectrophysiological mechanisms behind persAF would be helpful for the validation of the suggested method, such as in (i) computational intracardiac models that simulate both atrial electrical activity and ablation procedures during AF [61] and; (ii) prospective studies using the suggested method in the identification of ablation targets during substrate mapping. Additionally, we acknowledge that the simplistic and non-physiologic rationale behind the counting of the number of fractionated deflections as performed by the CARTO algorithm might also impose some limitations. This, however, deserves a dedicated investigation and is out of the scope of the present work.

The non-stationarity of AEGs collected during persAF makes the idealization of RQA-parameter choosing impractical. We believe, however, that the investigation on the temporal behavior of AEGs requires a more dedicated analysis and is out of the scope of the present work. Additionally, considering 
that most devices used to guide AF ablation still consider sequential point-by-point bipolar AEGs limited to $8 \mathrm{~s}$ duration, we believe that the results found in the present work are relevant and timely, as they can be applied with the currently available technology.

\section{Conclusions}

In the present work, we characterized the dynamics of atrial tissue activations from AEGs collected during persAF using RPs and RQA-based variables. We have shown that all features are sensitive to important electrophysiologic characteristics of the atrial tissue, and they were able to significantly discriminate normal AEGs from fractionated ones. Additionally, a high residual determinism was found in the RPs from fractionated AEGs, which could be related to unstable periodic orbits in a chaotic motion. This implies that the spatiotemporal dynamics of persAF mechanisms is not necessarily associated to a random structure. On the contrary, even the seemingly turbulent activations in fractionated AEGs are related to deterministic mechanisms of activation that could be a result of AF drivers that drift over the LA, with preferred locations. Therefore, RQA could be potentially used as biological markers to help with the characterization of the atrial substrate during the identification of targets for persAF ablation.

\section{Supplementary materials}

See supplementary material for the details of the clinical characteristics of the study subjects and for illustration of the ROC curves using the CARTO criteria as the reference for AEG classification and the RQA variables as discriminators.

\section{Acknowledgements}

The authors thank Prof. Takashi Yoneyama, Prof. Eduardo T. Costa and Dr. Fernanda Q. Rossi for their valuable suggestions. 


\section{Funding}

The work reported in this paper was supported by the NIHR Leicester Biomedical Research Centre. DCS receives CNPq financial support (449467/2014-7 and 305616/2016-1). TPA received research grants from Coordenação de Aperfeiçoamento de Pessoal de Nível Superior (CAPES, Brazil) and Fundação de Amparo à Pesquisa do Estado de São Paulo (FAPESP, n. 2017/00319-8).

\section{Conflict of Interest}

Prof. G. A. Ng has received a research fellowship from St. Jude Medical and speaker fees and honoraria from Biosense Webster. All other authors have reported that they have no relationships relevant to the contents of this paper to disclose. 


\section{References}

[1] J. P. Zbilut, N. Thomasson, C. L. Webber. Recurrence quantification analysis as a tool for nonlinear exploration of nonstationary cardiac signals. Medical Engineering \& Physics 24, 53-60 (2002).

[2] N. Marwan, N. Wessel, U. Meyerfeldt, A. Schirdewan, J. Kurths. Recurrence-plot-based measures of complexity and their application to heart-rate-variability data. Phys Rev E Stat Nonlin Soft Matter Phys 66, $026702(2002)$.

[3] N. Marwan, M. Carmen Romano, M. Thiel, J. Kurths. Recurrence plots for the analysis of complex systems. Physics Reports 438, 237-329 (2007).

[4] U. R. Acharya, H. Fujita, V. K. Sudarshan, D. N. Ghista, W. J. E. Lim, J. E. Koh. Automated Prediction of Sudden Cardiac Death Risk Using Kolmogorov Complexity and Recurrence Quantification Analysis Features Extracted from HRV Signals. IEEE International Conference on Systems, Man, and Cybernetics, 1110-5 (2015).

[5] H. Arce, A. Fuentes, G. H. González. Recurrence Analysis of Cardiac Restitution in Human Ventricle.

In: Webber JC, Ioana C, Marwan N, editors. Recurrence Plots and Their Quantifications: Expanding Horizons. Springer Proceedings in Physics: Springer, Cham; 2016.

[6] M. Tang, C. Q. Chang, P. C. Fung, K. T. Chau, F. H. Y. Chan. An Improved Method for Discriminating ECG Signals using Typical Nonlinear Dynamic Parameters and Recurrence Quantification Analysis in Cardiac Disease Therapy. 2005 IEEE Engineering in Medicine and Biology 27th Annual Conference, 2459-62 (2005).

[7] H. Yang. Multiscale recurrence quantification analysis of spatial cardiac vectorcardiogram signals. IEEE Trans Biomed Eng 58, 339-47 (2011). 
[8] D. Prichard, J. Theiler. Generalized redundancies for time series analysis. Physica D: Nonlinear Phenomena 84, 476-93 (1995).

[9] P. Faure, H. Korn. A new method to estimate the Kolmogorov entropy from recurrence plots: its application to neuronal signals. Physica D: Nonlinear Phenomena 122, 265-79 (1998).

[10] D. C. Soriano, R. Suyama, R. Attux. Blind extraction of chaotic sources from mixtures with stochastic signals based on recurrence quantification analysis. Digital Signal Processing 21, 417-26 (2011).

[11] D. C. Soriano, R. Suyama, R. A. Ando, R. Attux, L. T. Duarte. Blind source separation in the context of deterministic signals. In: Eisencraft M, Attux R, Suyama R, editors. Chaotic Signals in Digital Communications: CRC Press; 2013. p. 325-48.

[12] M. Mohebbi, H. Ghassemian, B. M. Asl. Structures of the Recurrence Plot of Heart Rate Variability Signal as a Tool for Predicting the Onset of Paroxysmal Atrial Fibrillation. Journal of Medical Signals and Sensors 1, 113-21 (2011).

[13] J. Schlenker, V. Socha, L. Riedlbauchová, T. Nedělka, A. Schlenker, V. Potočková, Š. Malá, P. Kutílek. Recurrence plot of heart rate variability signal in patients with vasovagal syncopes. Biomedical Signal Processing and Control 25, 1-11 (2016).

[14] A. Garfinkel, P. S. Chen, D. O. Walter, H. S. Karagueuzian, B. Kogan, S. J. Evans, M. Karpoukhin, C. Hwang, T. Uchida, et al. Quasiperiodicity and chaos in cardiac fibrillation. J Clin Invest 99, 305-14 (1997).

[15] J. N. Weiss, A. Garfinkel, H. S. Karagueuzian, Z. Qu, P. S. Chen. Chaos and the transition to ventricular fibrillation: a new approach to antiarrhythmic drug evaluation. Circulation 99, 2819-26 (1999). 
[16] F. X. Witkowski, K. M. Kavanagh, P. A. Penkoske, R. Plonsey, M. L. Spano, W. L. Ditto, D. T. Kaplan. Evidence for determinism in ventricular fibrillation. Physical Review Letters 75, 1230-3 (1995).

[17] A. L. Goldberger, V. Bhargava, B. J. West, A. J. Mandell. Some observations on the question: Is ventricular fibrillation “chaos”? Physica D: Nonlinear Phenomena 19, 282-9 (1986).

[18] D. T. Kaplan, R. J. Cohen. Is fibrillation chaos? Circ Res 67, 886-92 (1990).

[19] M. Small, D. Yu, R. G. Harrison, C. Robertson, G. Clegg, M. Holzer, F. Sterz. Deterministic nonlinearity in ventricular fibrillation. Chaos 10, 268-77 (2000).

[20] H. Calkins, G. Hindricks, R. Cappato, Y. H. Kim, E. B. Saad, L. Aguinaga, J. G. Akar, V. Badhwar, J. Brugada, et al. 2017 HRS/EHRA/ECAS/APHRS/SOLAECE expert consensus statement on catheter and surgical ablation of atrial fibrillation. Heart Rhythm 14, e275-e444 (2017).

[21] M. Haissaguerre, P. Jais, D. C. Shah, A. Takahashi, M. Hocini, G. Quiniou, S. Garrigue, A. Le Mouroux, P. Le Metayer, et al. Spontaneous initiation of atrial fibrillation by ectopic beats originating in the pulmonary veins. N Engl J Med 339, 659-66 (1998).

[22] J. M. T. de Bakker, F. H. M. Wittkampf. The Pathophysiologic Basis of Fractionated and Complex Electrograms and the Impact of Recording Techniques on Their Detection and Interpretation. Circ Arrhythm Electrophysiol 3, 204-13 (2010).

[23] K. T. Konings, C. J. Kirchhof, J. R. Smeets, H. J. Wellens, O. C. Penn, M. A. Allessie. High-density mapping of electrically induced atrial fibrillation in humans. Circulation 89, 1665-80 (1994).

[24] J. Jalife, O. Berenfeld, M. Mansour. Mother rotors and fibrillatory conduction: a mechanism of atrial fibrillation. Cardiovasc Res 54, 204-16 (2002). 
[25] N. de Groot, L. van der Does, A. Yaksh, E. Lanters, C. Teuwen, P. Knops, P. van de Woestijne, J. Bekkers, C. Kik, et al. Direct Proof of Endo-Epicardial Asynchrony of the Atrial Wall During Atrial Fibrillation in Humans. Circ Arrhythm Electrophysiol 9, (2016).

[26] S. M. Narayan, D. E. Krummen, K. Shivkumar, P. Clopton, W. J. Rappel, J. M. Miller. Treatment of atrial fibrillation by the ablation of localized sources: CONFIRM (Conventional Ablation for Atrial Fibrillation With or Without Focal Impulse and Rotor Modulation) trial. J Am Coll Cardiol 60, 628-36 (2012).

[27] K. Nademanee, J. McKenzie, E. Kosar, M. Schwab, B. Sunsaneewitayakul, T. Vasavakul, C. Khunnawat, T. Ngarmukos. A new approach for catheter ablation of atrial fibrillation: Mapping of the electrophysiologic substrate. J Am Coll Cardiol 43, 2044-53 (2004).

[28] R. M. Hayward, G. A. Upadhyay, T. Mela, P. T. Ellinor, C. D. Barrett, E. K. Heist, A. Verma, N. K. Choudhry, J. P. Singh. Pulmonary vein isolation with complex fractionated atrial electrogram ablation for paroxysmal and nonparoxysmal atrial fibrillation: A meta-analysis. Heart Rhythm 8, 994-1000 (2011).

[29] T. P. Almeida, G. S. Chu, X. Li, N. Dastagir, J. H. Tuan, P. J. Stafford, F. S. Schlindwein, G. A. Ng. Atrial Electrogram Fractionation Distribution before and after Pulmonary Vein Isolation in Human Persistent Atrial Fibrillation-A Retrospective Multivariate Statistical Analysis. Front Physiol 8, (2017).

[30] S. Dixit, F. E. Marchlinski, D. Lin, D. J. Callans, R. Bala, M. P. Riley, F. C. Garcia, M. D. Hutchinson, S. J. Ratcliffe, et al. Randomized ablation strategies for the treatment of persistent atrial fibrillation: RASTA study. Circ Arrhythm Electrophysiol 5, 287-94 (2012).

[31] A. Verma, C. Y. Jiang, T. R. Betts, J. Chen, I. Deisenhofer, R. Mantovan, L. Macle, C. A. Morillo, W. Haverkamp, et al. Approaches to catheter ablation for persistent atrial fibrillation. N Engl J Med 372, $1812-22(2015)$. 
[32] J. Vogler, S. Willems, A. Sultan, D. Schreiber, J. Luker, H. Servatius, B. Schaffer, J. Moser, B. A. Hoffmann, et al. Pulmonary Vein Isolation Versus Defragmentation: The CHASE-AF Clinical Trial. J Am Coll Cardiol 66, 2743-52 (2015).

[33] E. Buch, M. Share, R. Tung, P. Benharash, P. Sharma, J. Koneru, R. Mandapati, K. A. Ellenbogen, K. Shivkumar. Long-term clinical outcomes of focal impulse and rotor modulation for treatment of atrial fibrillation: A multicenter experience. Heart Rhythm 13, 636-41 (2016).

[34] C. Gianni, S. Mohanty, L. Di Biase, T. Metz, C. Trivedi, Y. Gokoglan, M. F. Gunes, R. Bai, A. AlAhmad, et al. Acute and early outcomes of focal impulse and rotor modulation (FIRM)-guided rotorsonly ablation in patients with nonparoxysmal atrial fibrillation. Heart Rhythm 13, 830-5 (2016).

[35] J. S. Steinberg, Y. Shah, A. Bhatt, T. Sichrovsky, A. Arshad, E. Hansinger, D. Musat. Focal impulse and rotor modulation: Acute procedural observations and extended clinical follow-up. Heart Rhythm 14, 192-7 (2017).

[36] R. Providência, P. D. Lambiase, N. Srinivasan, G. Ganesha Babu, K. Bronis, S. Ahsan, F. Z. Khan, A. W. C. Chow, E. Rowland, et al. Is There Still a Role for CFAE Ablation in Addition to Pulmonary Vein Isolation in Patients with Paroxysmal and Persistent Atrial Fibrillation? A Meta-Analysis of 1,415 Patients. Circulation: Arrhythmia and Electrophysiology, (2015).

[37] T. P. Almeida, G. S. Chu, J. L. Salinet, F. J. Vanheusden, X. Li, J. H. Tuan, P. J. Stafford, G. A. Ng, F. S. Schlindwein. Minimizing discordances in automated classification of fractionated electrograms in human persistent atrial fibrillation. Medical \& Biological Engineering \& Computing 54, 1695-706 (2016).

[38] T. P. Almeida, G. S. Chu, M. J. Bell, X. Li, J. L. Salinet, N. Dastagir, J. H. Tuan, P. J. Stafford, G. André $\mathrm{Ng}$, et al. The temporal behavior and consistency of bipolar atrial electrograms in human persistent atrial fibrillation. Medical \& Biological Engineering \& Computing, In press (2017). 
[39] M. Haissaguerre, M. Hocini, A. Denis, A. J. Shah, Y. Komatsu, S. Yamashita, M. Daly, S. Amraoui, S. Zellerhoff, et al. Driver Domains in Persistent Atrial Fibrillation. Circulation, (2014).

[40] J. Salinet, F. S. Schlindwein, P. Stafford, T. P. Almeida, X. Li, F. J. Vanheusden, M. S. Guillem, G. A. Ng. Propagation of meandering rotors surrounded by areas of high dominant frequency in persistent atrial fibrillation. Heart Rhythm 14, 1269-78 (2017).

[41] J. L. Salinet, J. H. Tuan, A. J. Sandilands, P. J. Stafford, F. S. Schlindwein, G. André Ng. Distinctive Patterns of Dominant Frequency Trajectory Behavior in Drug-Refractory Persistent Atrial Fibrillation. J Cardiovasc Electrophysiol 25, 371-9 (2013).

[42] C. D. Marcotte, R. O. Grigoriev. Dynamical mechanism of atrial fibrillation: A topological approach. Chaos 27, 093936 (2017).

[43] J. L. Wells, R. B. Karp, N. T. Kouchoukos, W. A. H. Maclean, T. N. James, A. L. Waldo. Characterization of Atrial-Fibrillation in Man - Studies Following Open-Heart Surgery. Pace-Pacing and Clinical Electrophysiology 1, 426-38 (1978).

[44] F. Censi, V. Barbaro, P. Bartolini, G. Calcagnini, A. Michelucci, G. F. Gensini, S. Cerutti. Recurrent patterns of atrial depolarization during atrial fibrillation assessed by recurrence plot quantification. Ann Biomed Eng 28, 61-70 (2000).

[45] N. Navoret, S. Jacquir, G. Laurent, S. Binczak. Detection of Complex Fractionated Atrial Electrograms Using Recurrence Quantification Analysis. Ieee Transactions on Biomedical Engineering 60, 1975-82 (2013).

[46] J. Ng, D. Gordon, R. S. Passman, B. P. Knight, R. Arora, J. J. Goldberger. Electrogram morphology recurrence patterns during atrial fibrillation. Heart Rhythm 11, 2027-34 (2014). 
[47] D. Gordon, J. J. Goldberger, R. Arora, G. L. Aistrup, J. Ng. Searching for "order" in atrial fibrillation using electrogram morphology recurrence plots. Computers in Biology and Medicine 65, 220-8 (2015).

[48] S. Zeemering, P. Bonizzi, B. Maesen, R. Peeters, U. Schotten. Recurrence quantification analysis applied to spatiotemporal pattern analysis in high-density mapping of human atrial fibrillation. Conf Proc IEEE Eng Med Biol Soc 2015, 7704-7 (2015).

[49] J. P. Hummel, A. Baher, B. Buck, M. Fanarjian, C. L. Webber, J. G. Akar. A method for quantifying recurrent patterns of local wavefront direction during atrial fibrillation. Computers in Biology and Medicine 89, 497-504 (2017).

[50] J. Tuan, M. Jeilan, S. Kundu, W. Nicolson, I. Chung, P. J. Stafford, G. A. Ng. Regional fractionation and dominant frequency in persistent atrial fibrillation: effects of left atrial ablation and evidence of spatial relationship. Europace 13, 1550-6 (2011).

[51] C. Schilling. Analysis of Atrial Electrograms. KIT Scientific Publishing: Karlsruhe Institute of Technology; 2012.

[52] C. L. Webber, J. P. Zbilut. Recurrence quantifications: feature extractions from recurrence plots. International Journal of Bifurcation and Chaos 17, 3467-75 (2007).

[53] C. L. Webber, J. P. Zbilut. Dynamical assessment of physiological systems and states using recurrence plot strategies. Journal of Applied Physiology 76, 965-73 (1994).

[54] N. Marwan. How to avoid potential pitfalls in recurrence plot based data analysis. International Journal of Bifurcation and Chaos 21, 1003-17 (2011).

[55] G. K. Moe, J. A. Abildskov. Atrial fibrillation as a self-sustaining arrhythmia independent of focal discharge. Am Heart J 58, 59-70 (1959). 
[56] J. L. Salinet, M. S. Guillem, T. P. Almeida, X. Li, G. Goroso, G. S. Chu, G. A. Ng, F. S. Schlindwein. Drifting rotor prevalence is associated with dominant frequency reduction after persistent atrial fibrillation ablation. 2015 Computing in Cardiology Conference (CinC), 269-72 (2015).

[57] M. Martínez-Iniesta, J. Ródenas, R. Alcaraz, J. J. Rieta. Waveform Integrity in Atrial Fibrillation: The Forgotten Issue of Cardiac Electrophysiology. Ann Biomed Eng 45, 1890-907 (2017).

[58] M. T. Rosenstein, J. J. Collins. Visualizing the effects of filtering chaotic signals. Computers \& Graphics 18, 587-92 (1994).

[59] H. Cochet, R. Dubois, S. Yamashita, N. Al Jefairi, B. Berte, J.-M. Sellal, D. Hooks, A. Frontera, S. Amraoui, et al. Relationship Between Fibrosis Detected on Late Gadolinium-Enhanced CMR and ReEntrant Activity Assessed With ECGI in Human Persistent Atrial Fibrillation. JACC: Clinical Electrophysiology, (2017).

[60] A. Frontera, M. Takigawa, R. Martin, N. Thompson, G. Cheniti, G. Massoullié, J. Duchateau, J. Y. Wielandts, E. Teijeira, et al. Electrogram signature of specific activation patterns: Analysis of atrial tachycardias at high-density endocardial mapping. Heart Rhythm, (2017).

[61] M. W. Krueger, W. H. Schulze, K. S. Rhode, R. Razavi, G. Seemann, O. Dossel. Towards personalized clinical in-silico modeling of atrial anatomy and electrophysiology. Medical \& Biological Engineering \& Computing 51, 1251-60 (2013). 


\section{Tables}

Table 1. The area under the ROC curves (AUROC) for the discrimination between normal vs. fractionated AEGs considering RR as the discriminator. RR was calculated for eleven different values of $\varepsilon$, varying from $0.5 \%$ to $10 \%$ of the maximum phase space distance.

\begin{tabular}{cccccccccccc}
\hline \% of max phase space distance & $\mathbf{0 . 5 \%}$ & $\mathbf{1 \%}$ & $\mathbf{2 \%}$ & $\mathbf{3 \%}$ & $\mathbf{4 \%}$ & $\mathbf{5 \%}$ & $\mathbf{6 \%}$ & $\mathbf{7 \%}$ & $\mathbf{8 \%}$ & $\mathbf{9 \%}$ & $\mathbf{1 0 \%}$ \\
\hline AUROC & 0.717 & 0.714 & $\mathbf{0 . 7 0 2}$ & 0.690 & 0.678 & 0.667 & 0.656 & 0.646 & 0.637 & 0.627 & 0.618 \\
\hline
\end{tabular}

The highlighted value indicates the chosen threshold for $\varepsilon$ used for the remaining parts of the work.

Table 2. The AUROC for the discrimination between normal vs. fractionated AEGs considering DET and LAM as the discriminators, calculated with different minimum line lengths.

\begin{tabular}{|c|c|c|c|c|c|c|c|c|c|c|c|c|c|c|c|c|c|c|c|}
\hline Min line & & & & & & & & & & & & & & & & & & & \\
\hline length & 2 & 3 & 4 & 5 & 6 & 7 & 8 & 9 & 10 & 11 & 12 & 13 & 14 & 15 & 16 & 17 & 18 & 19 & 20 \\
\hline DET & 0.713 & 0.709 & 0.708 & 0.709 & 0.709 & 0.705 & 0.701 & 0.697 & 0.695 & 0.693 & 0.692 & 0.692 & 0.693 & 0.694 & 0.696 & 0.697 & 0.699 & 0.701 & 0.703 \\
\hline LAM & 0.717 & 0.713 & 0.713 & 0.715 & 0.717 & 0.717 & 0.716 & 0.715 & 0.714 & 0.714 & 0.715 & 0.714 & 0.715 & 0.715 & 0.715 & 0.715 & 0.716 & 0.717 & 0.717 \\
\hline
\end{tabular}

The highlighted values indicate the values chosen for DET and LAM used for the remaining parts of the work. 
Table 3. ROC curves for the AEG discrimination using the RQA-based features (mean $\pm \mathrm{SD}$ ).

\begin{tabular}{cccccccc}
\hline & RR & DET & L & ENTR & LAM & TT & ENTR_vert \\
\hline AUROC & $0.697 \pm 0.001$ & $0.709 \pm 0.001$ & $0.729 \pm 0.001$ & $0.693 \pm 0.001$ & $0.724 \pm 0.001$ & $0.716 \pm 0.001$ & $0.689 \pm 0.001$ \\
Operating Point & $0.076 \pm 0.001$ & $0.710 \pm 0.0001$ & $74.89 \pm 0.003$ & $2.85 \pm 0.05$ & $0.807 \pm 0.006$ & $11.02 \pm 0.003$ & $3.57 \pm 0.004$ \\
Sensitivity & $0.684 \pm 0.004$ & $0.710 \pm 0.001$ & $0.713 \pm 0.001$ & $0.679 \pm 0.027$ & $0.716 \pm 0.013$ & $0.684 \pm 0.001$ & $0.697 \pm 0.002$ \\
Specificity & $0.359 \pm 0.004$ & $0.369 \pm 0.001$ & $0.336 \pm 0.001$ & $0.361 \pm 0.023$ & $0.363 \pm 0.013$ & $0.336 \pm 0.001$ & $0.390 \pm 0.002$ \\
LOOCV hit rate & $64 \%$ & $66 \%$ & $69 \%$ & $62 \%$ & $64 \%$ & $67 \%$ & $63 \%$ \\
\hline
\end{tabular}




\section{Figure Titles and Captions:}

Figure 1. Illustration of automated AEG classification performed by algorithms embedded in commercial systems and types of AEGs. 3D atrial geometry representation for one patient, with the automated AEG classification algorithm performed by the CARTO (Biosense Webster, Diamond Bar, California) criteria. On the bottom part of the figure, the top trace refers to a segment of normal AEG (AEG 1), and the bottom trace refers to a fractionated AEG (AEG 2), both recorded from the LA endocardium. (Modified from [37]). LPV = left pulmonary veins; $\mathrm{MV}=$ mitral valve; RPV = right pulmonary veins.

Figure 2. A. Catheter ablation of an $\mathrm{AF}$ patient. The various computer screens show the 3D representation of the LA and online automated AEG classification created by the CARTO system to help

guide the ablation. B. A highlight of the $3 \mathrm{D}$ representation of the LA and the annotated AEG. C. AEG state space reconstruction following the Takens' theorem, time-delay embedding $(\tau)$ and embedding dimension $(d)$. D. Distance plot based on state space map, and the resulting RP after an adaptive threshold $\varepsilon$ was applied on the distance plot.

Figure 3. The definition of the parameters for recurrence analysis: $\varepsilon$ (A), and the minimum line length for the calculation of DET $(\mathbf{B})$ and LAM $(\mathbf{C})$.

Figure 4. The effect of SWT filter in the RQA. A. The RPs created for the same AEG, before (left) and after (right) the SWT filter. B. The probability distribution of diagonal lines for all AEGs, before (left) and after (right) the SWT filter. The KL divergence was calculated to compare the distributions.

Figure 5. Illustration of typical AEGs found in the present cohort - normal (left-hand side) and fractionated (right-hand side) - and their respective RPs. 
Figure 6. The RQA-based variables calculated from the AEGs classified as normal or fractionated by CARTO. A. The RR. B. Variables related to diagonal lines. C. Variables related to vertical lines. **** $\mathrm{P}<0.0001$.

Figure 7. The RQA-based variables calculated from the AEGs collected before and after PVI+RL. A. The RR. B. Variables related to diagonal lines. C. Variables related to vertical lines. $* * * * \mathrm{P}<0.0001$. 


\section{Automated AEG classification CARTO's criteria}

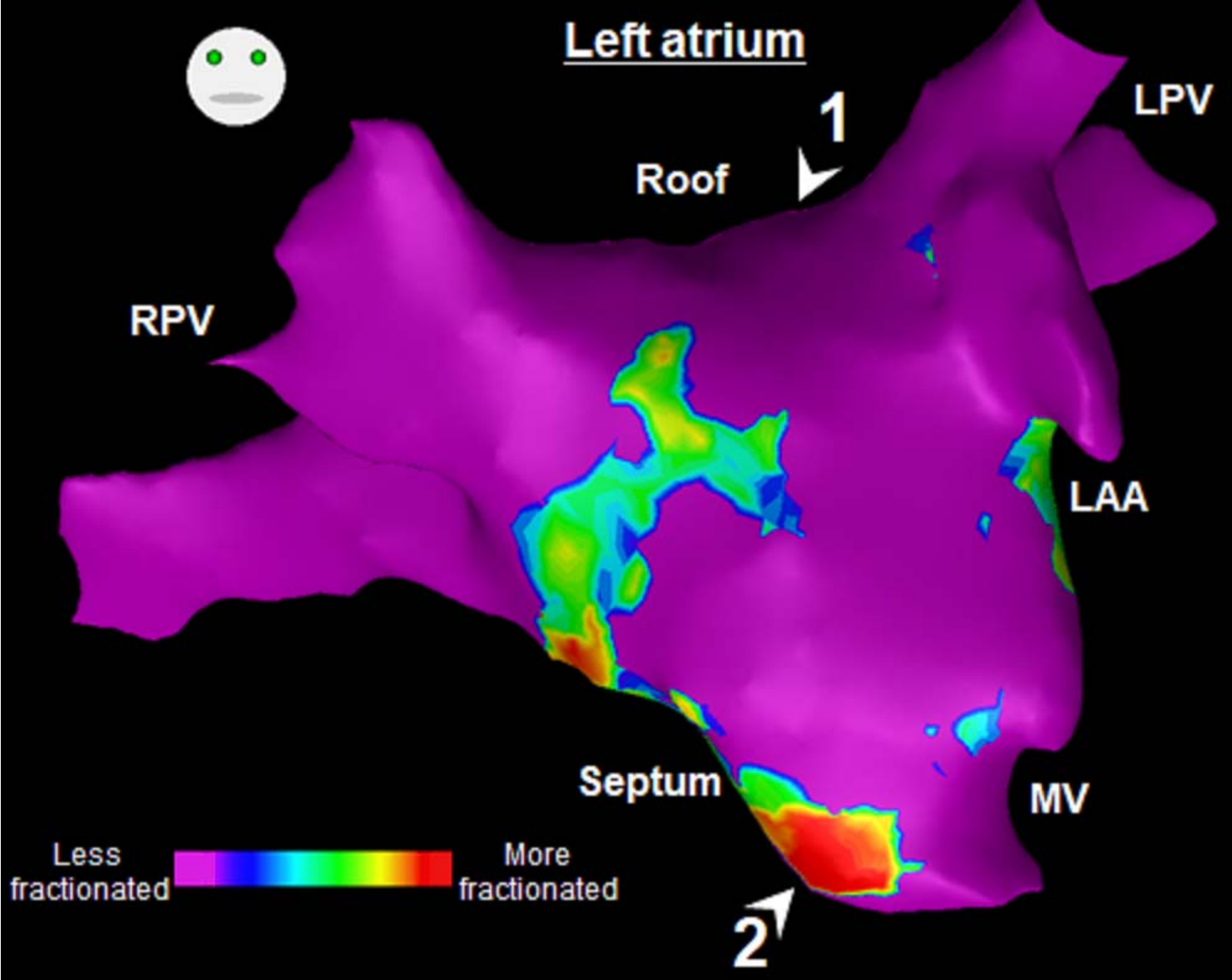

1. Normal AEG

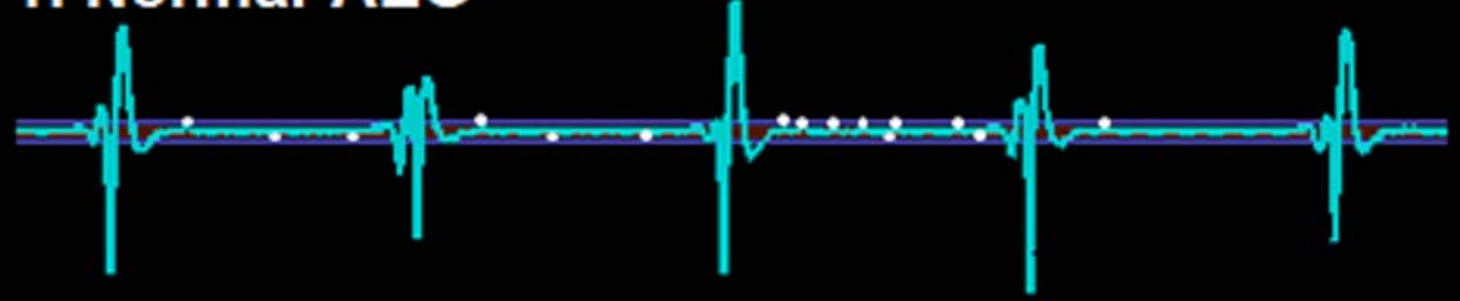

2. Fractionated AEG

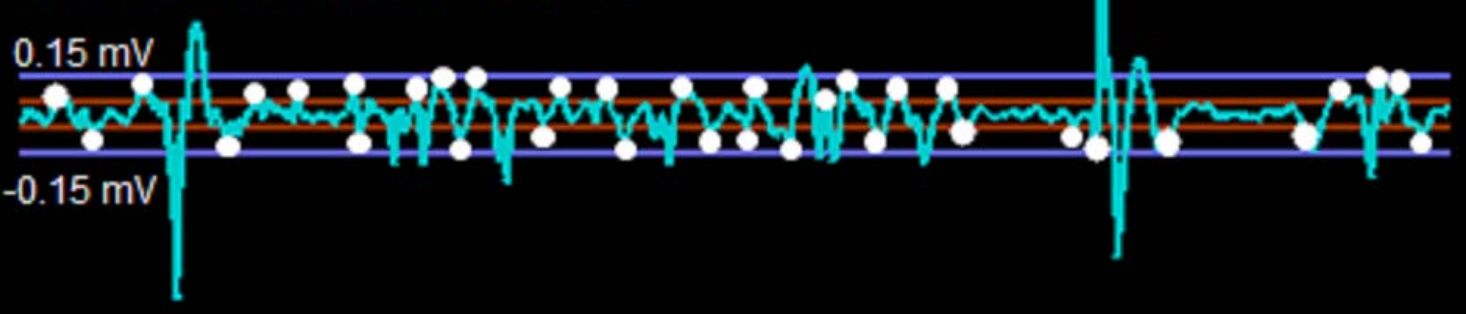


Figure 2

A. Electrophysiological study
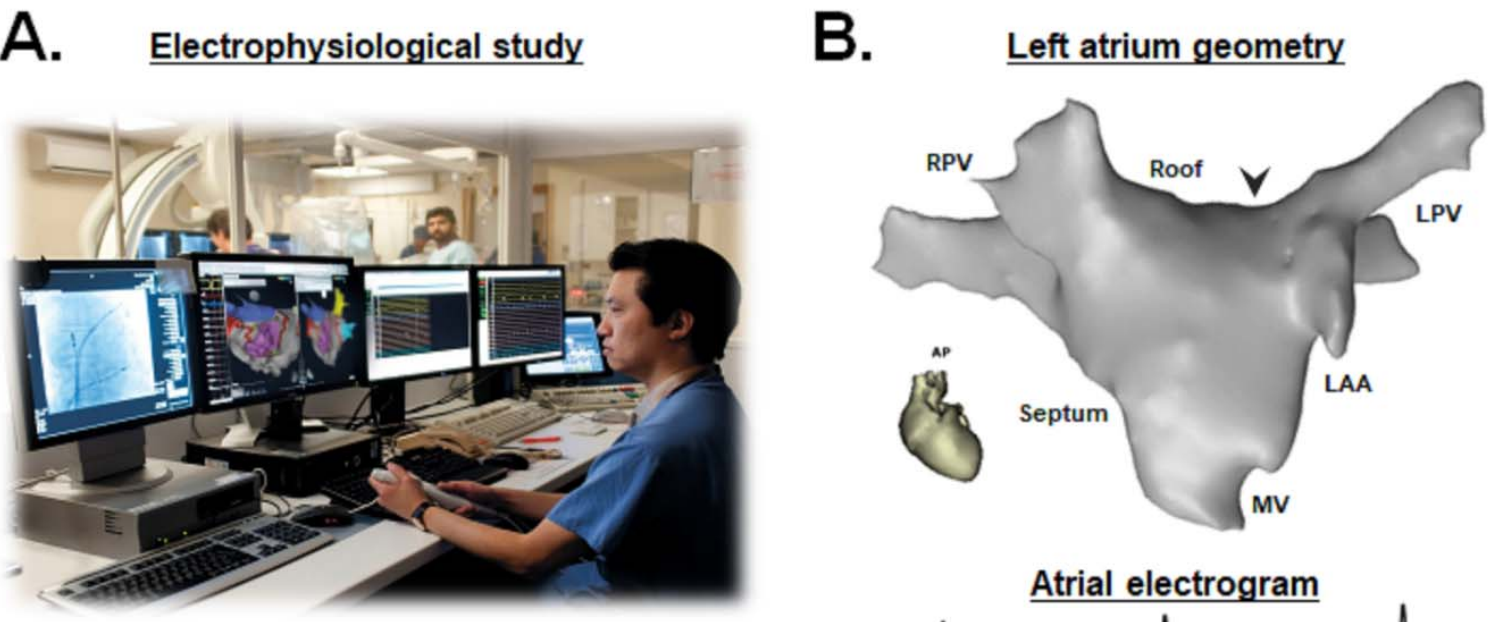

Atrial electrogram

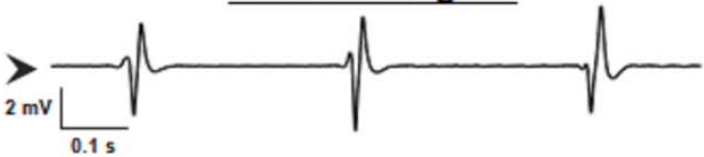

C. Mutual information
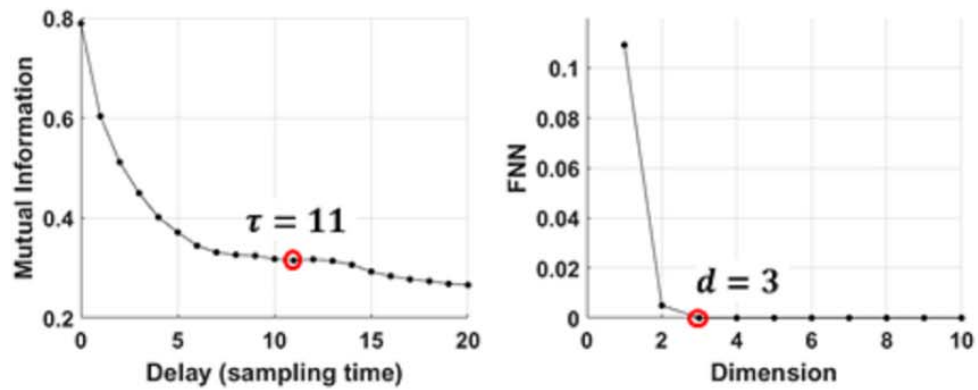

D.

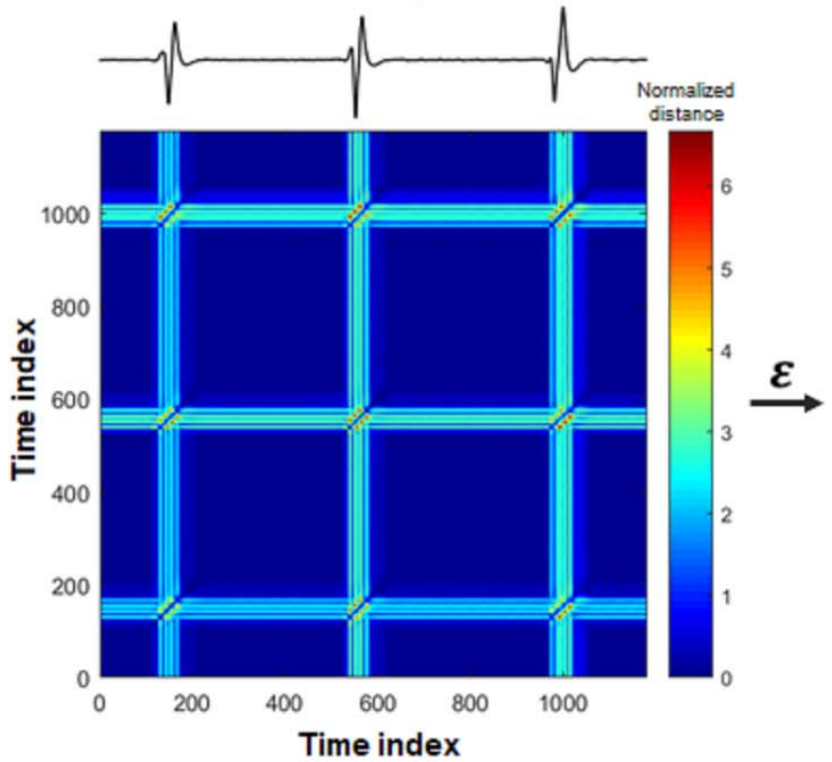

False nearest neighbor test

AEG time-delay embedding (state space plot)
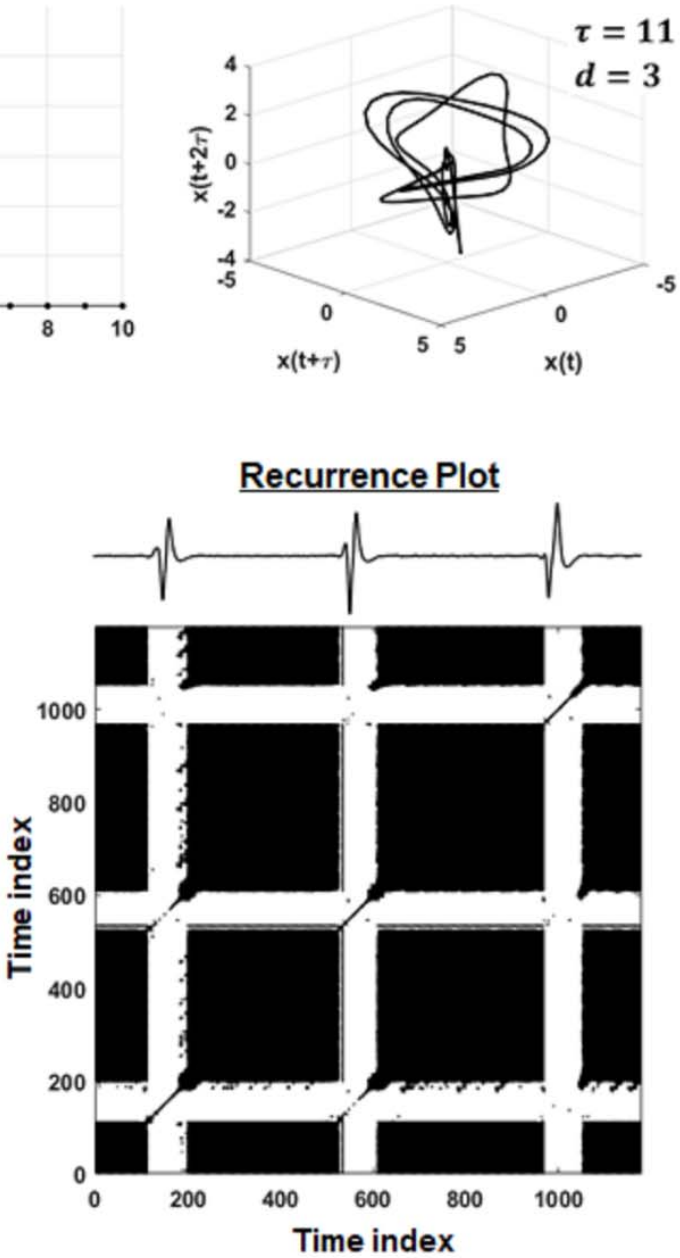
Figure 3

Almeida et al.

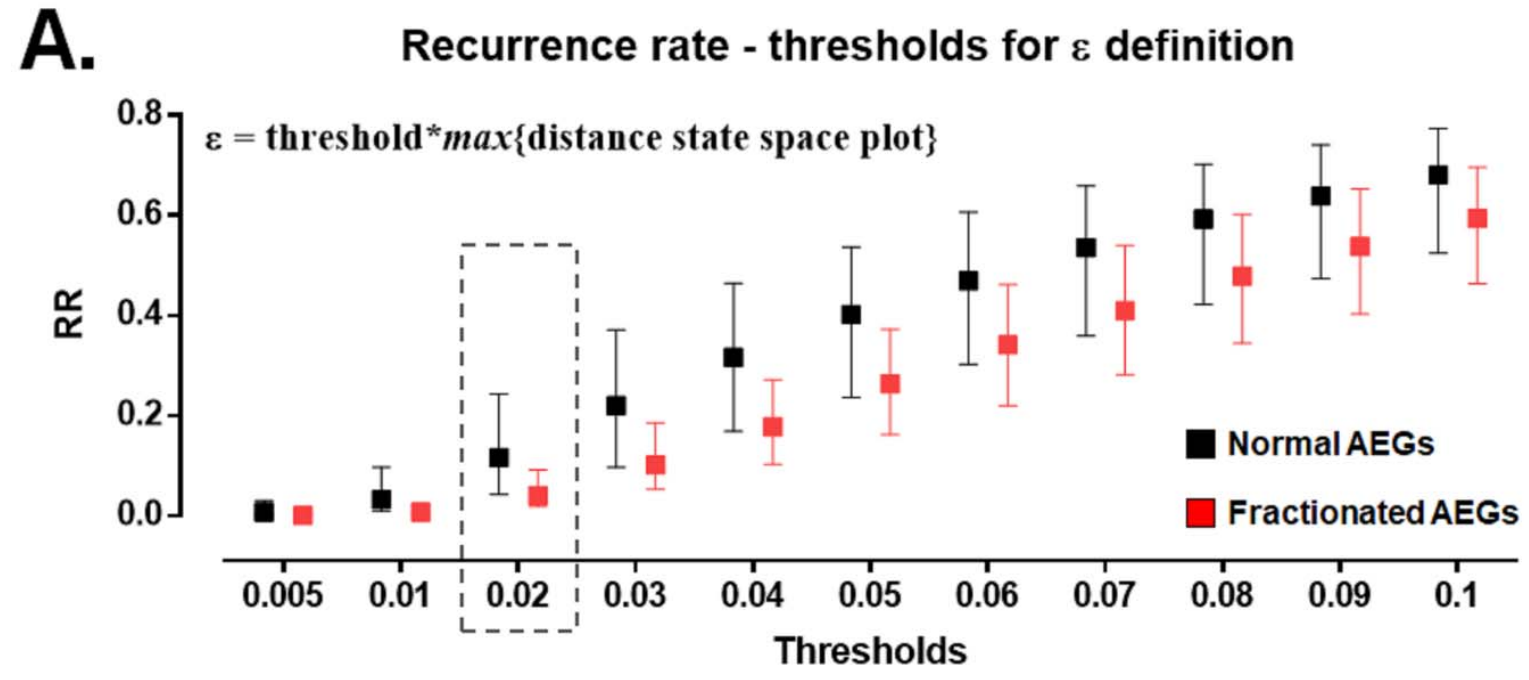

B.

Minimum diagonal line length for determinism
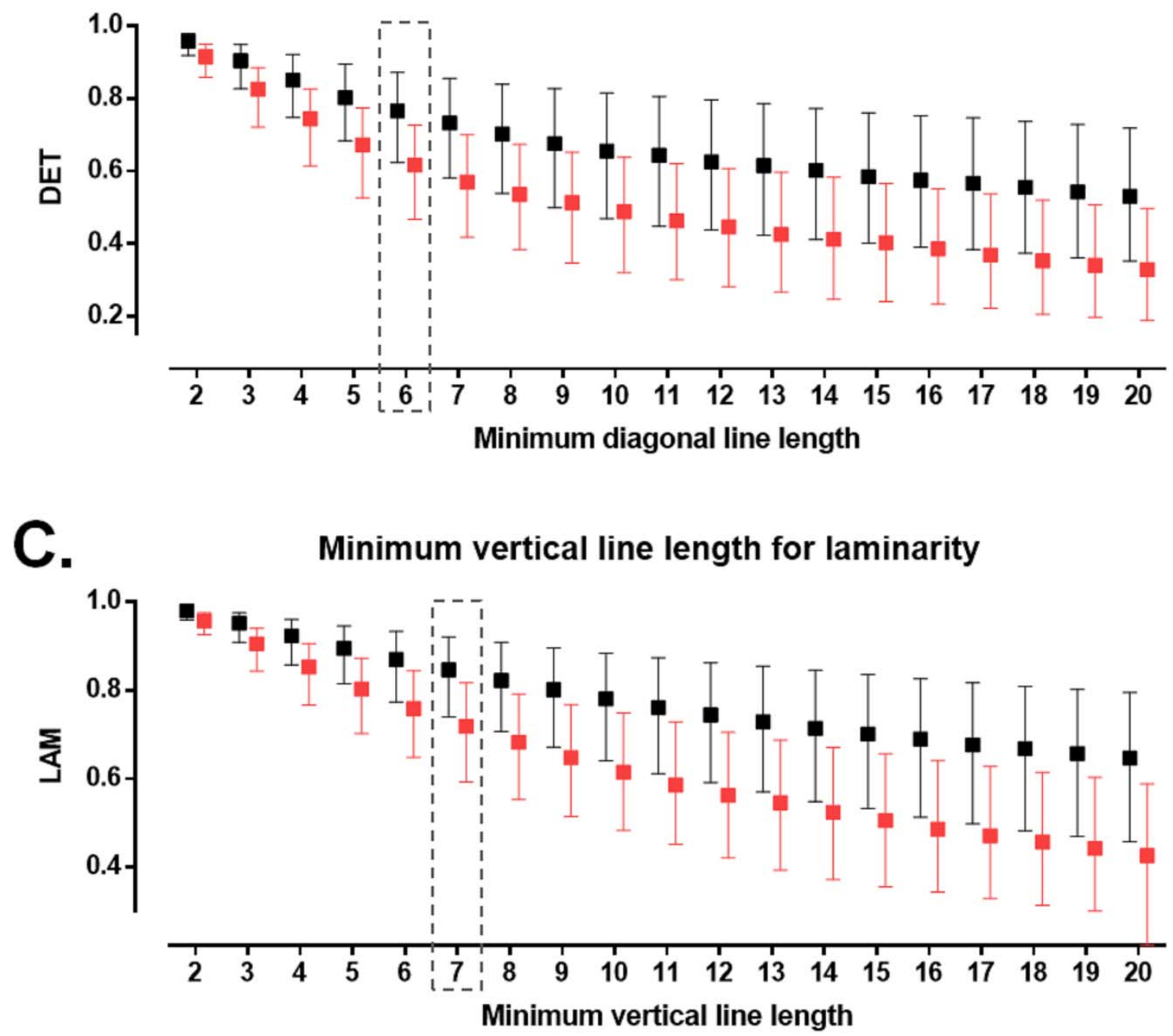
Figure 4
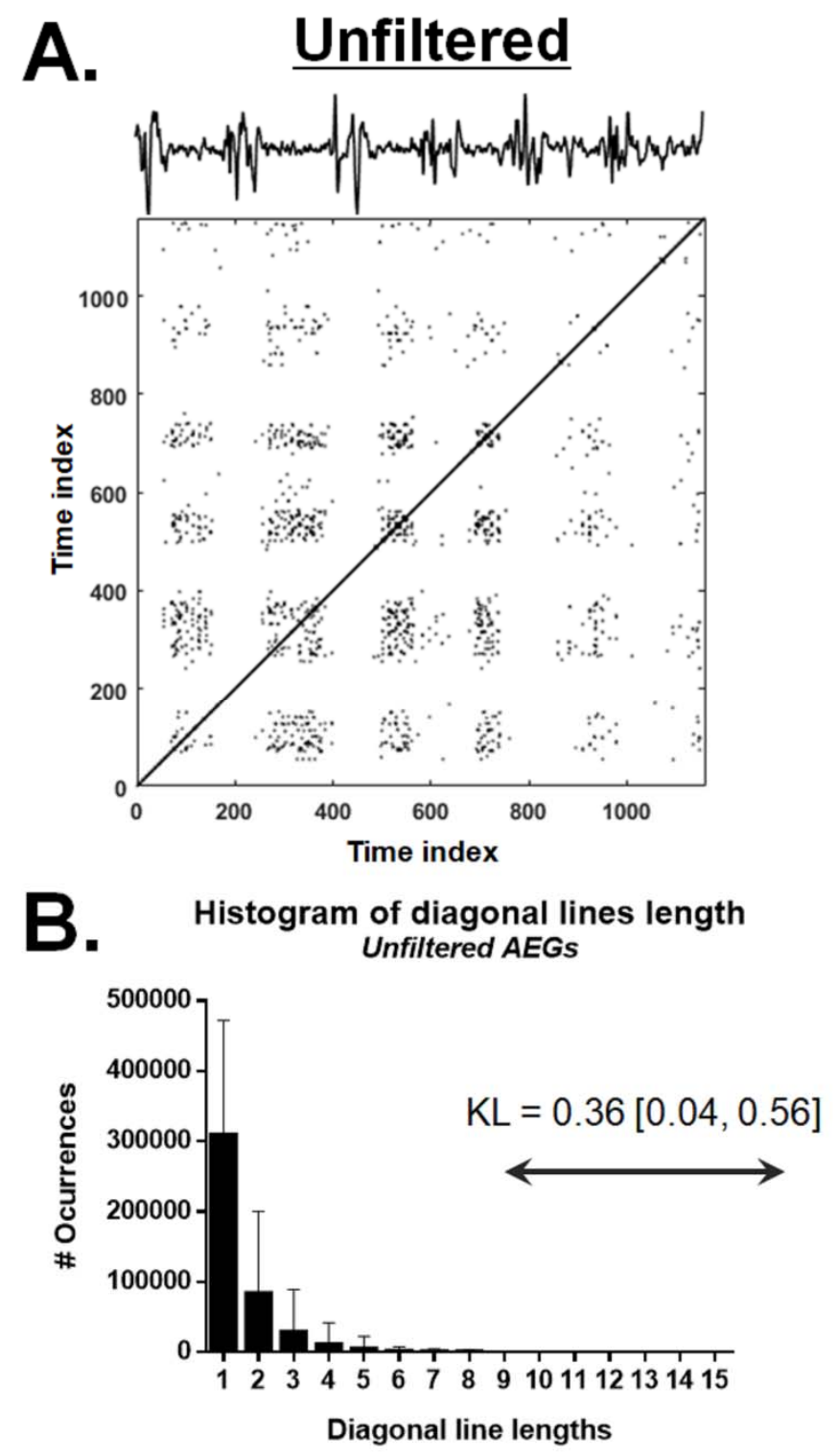
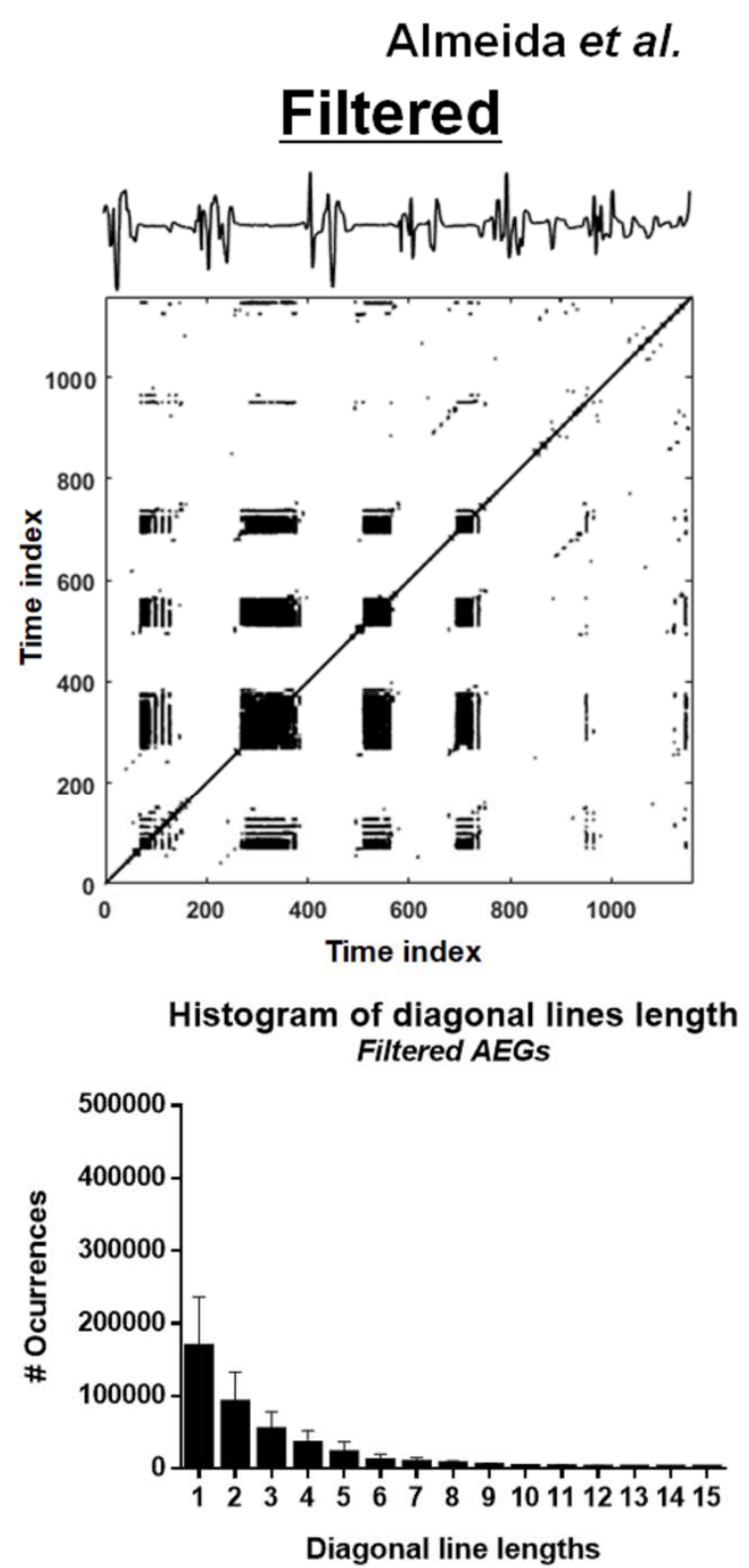
Figure 5

\section{Normal AEG}

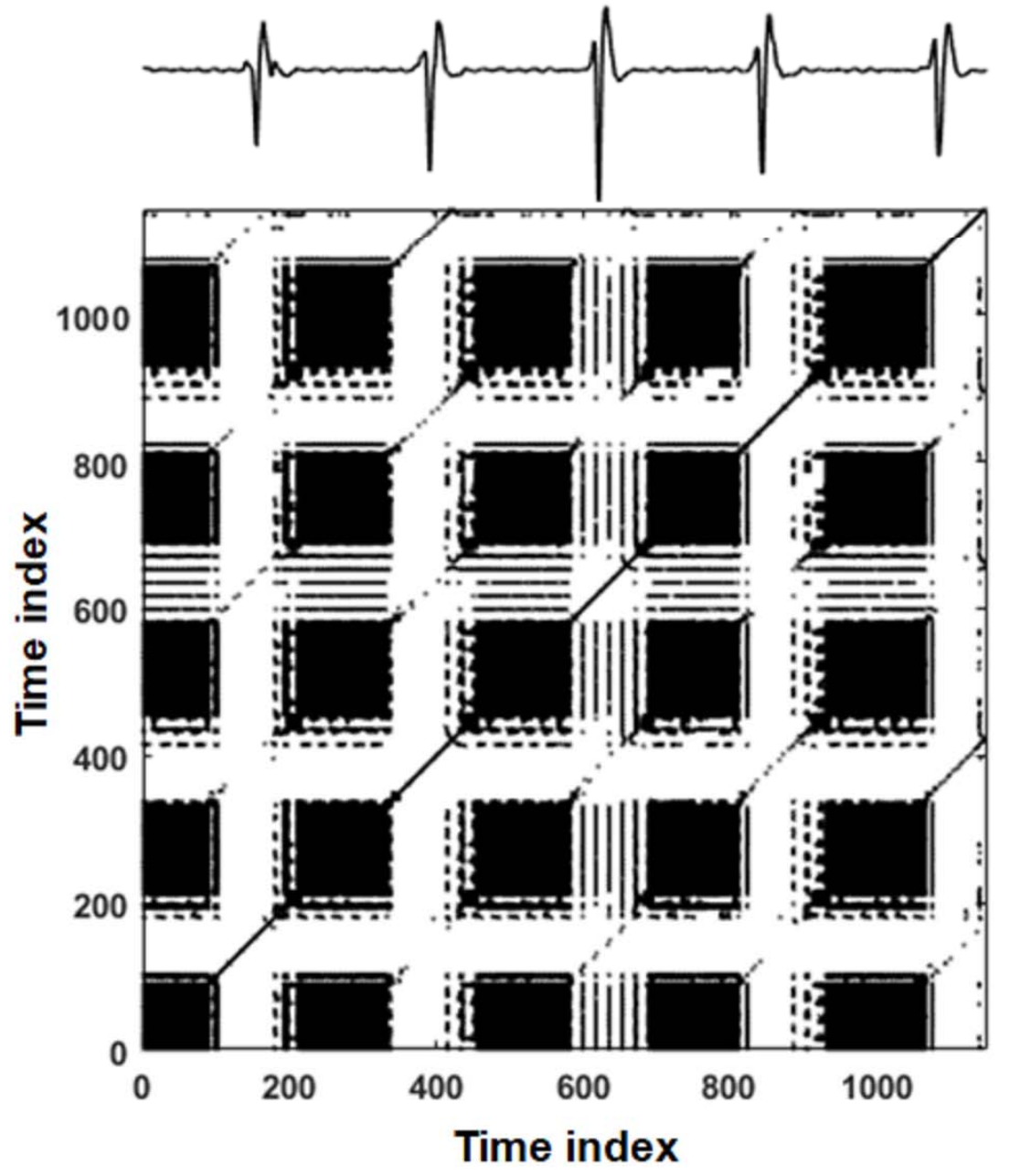

Almeida et al.

\section{Fractionated AEG}

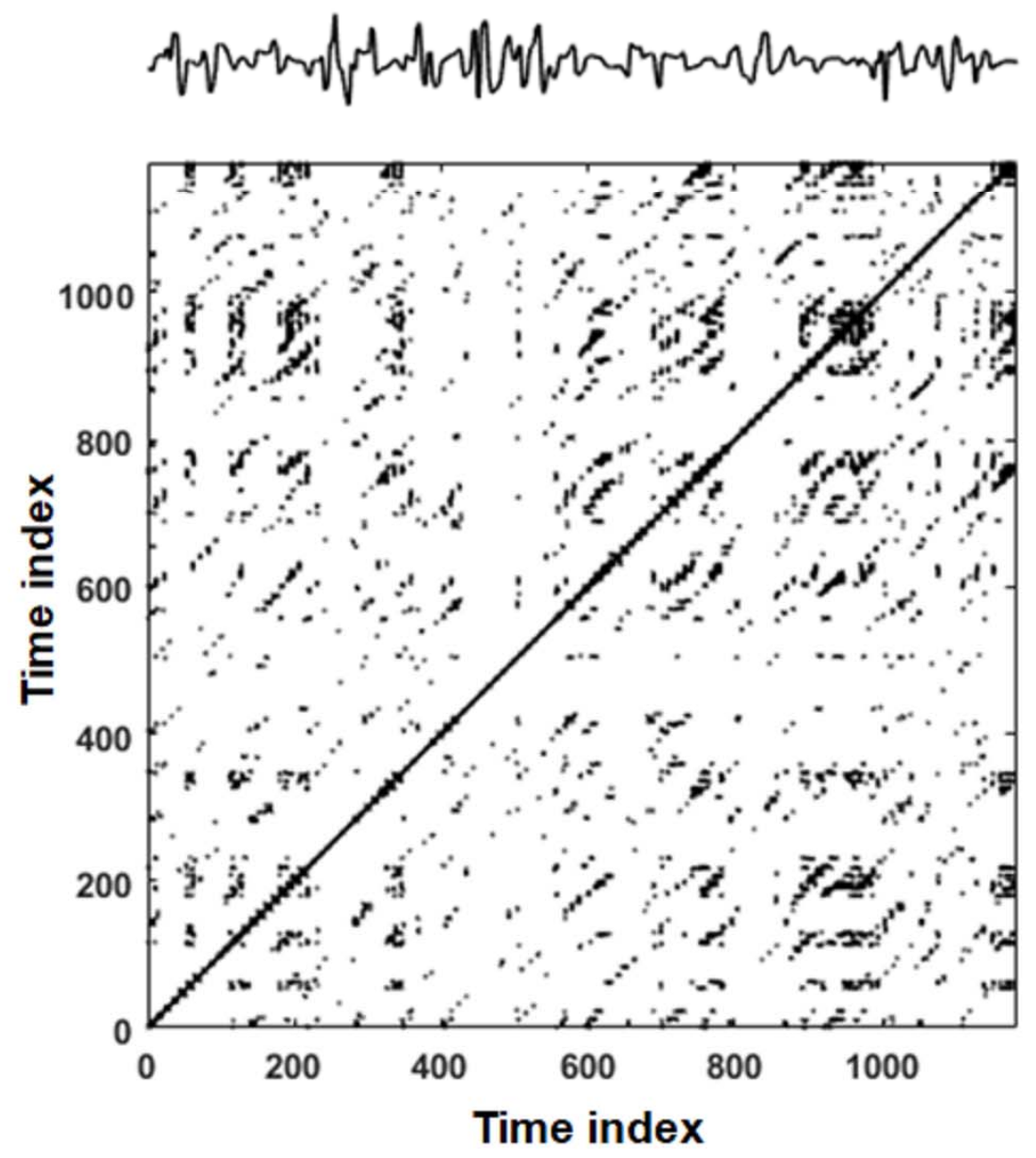




\section{Figure 6}

Almeida et al.

\section{RQA in normal vs. fractionated AEGs}

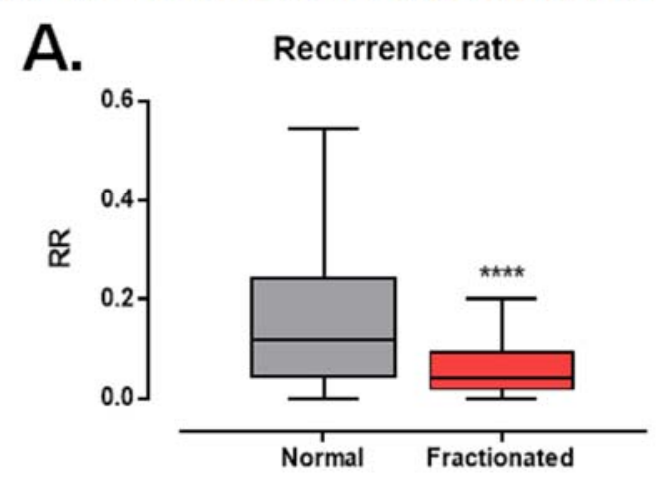

B.

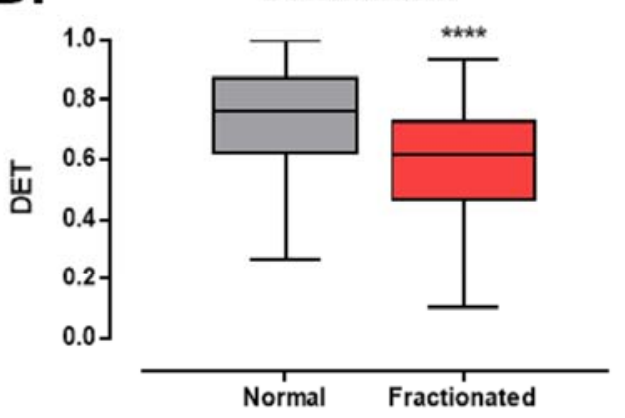

C.

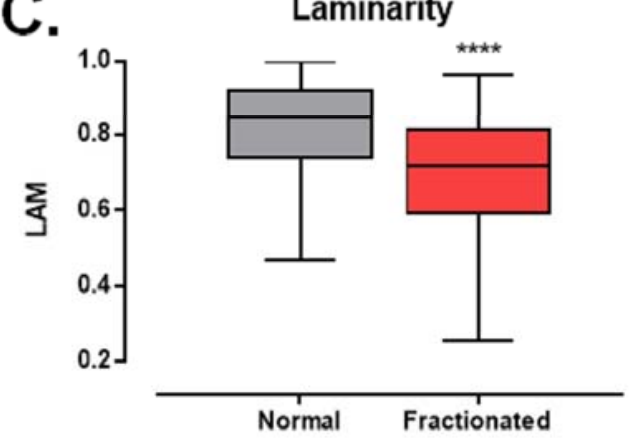

Average diagonal line length

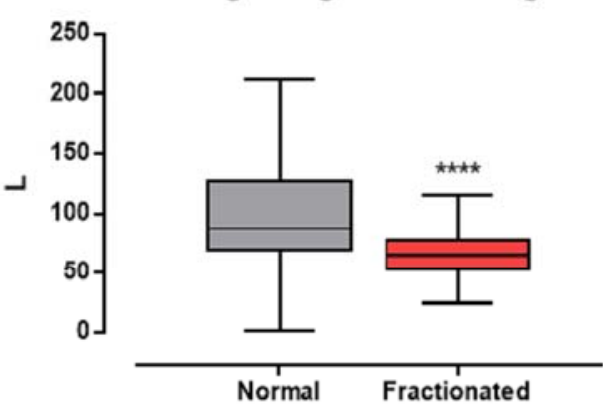

Trapping time

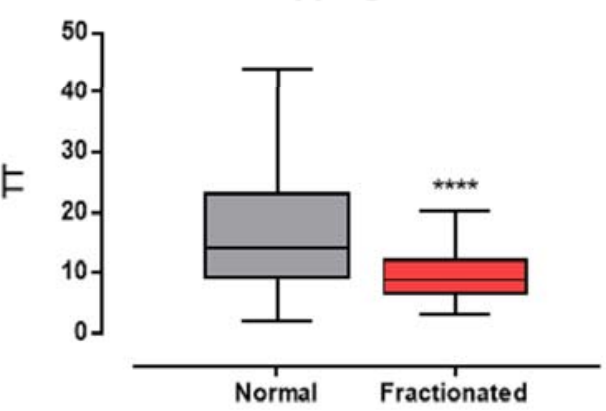

Entropy of diagonal lines

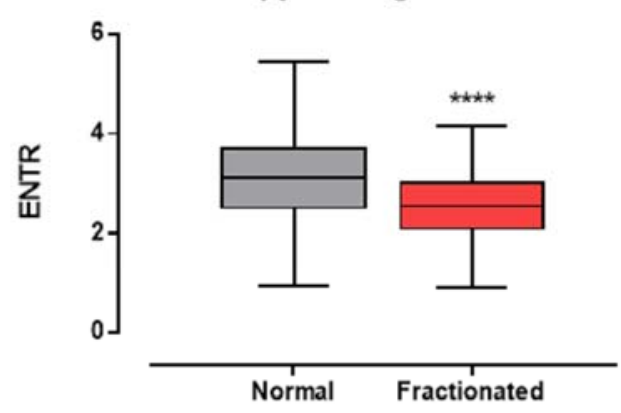

Entropy of vertical lines

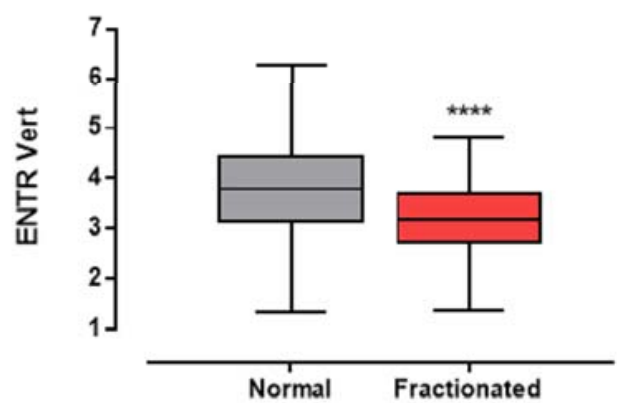


Figure 7

RQA before and after PVI+RL

A. Recurrence rate
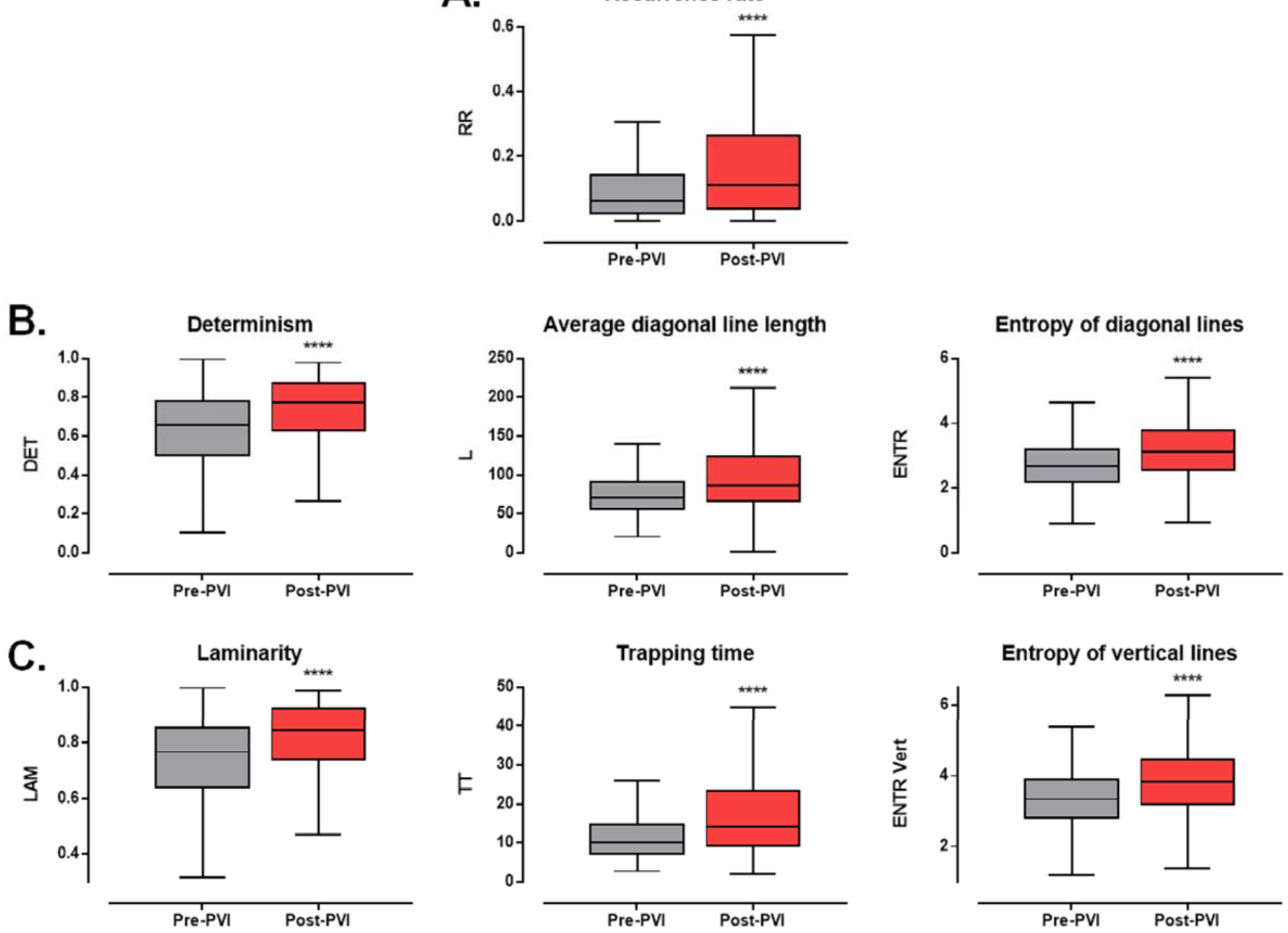